\title{
Corrections to flat-space particle dynamics arising from space granularity.
}

\author{
Luis F. Urrutia \\ Instituto de Ciencias Nucleares, Universidad Nacional Autónoma de México, \\ Circuito Exterior, C.U., 04510 México, D.F. urrutia@nucleares.unam.mx
}

Summary. The construction of effective Hamiltonians describing corrections to flat space particle dynamics arising from the granularity of space at very short distances is discussed in the framework of an heuristic approach to the semiclassical limit of loop quantum gravity. After some general motivation of the subject, a brief non-specialist introduction to the basic tools employed in the loop approach is presented. The heuristical semiclassical limit is subsequently defined and the application to the case of photons and spin $1 / 2$ fermions is described. The resulting modified Maxwell and Dirac Hamiltonians, leading in particular to Planck scale corrections in the energy-momentum relations, are presented. Alternative interpretations of the results and their limitations, together with other approaches are briefly discussed along the text. Three topics related to the above methods are reviewed: (1) The determination of bounds to the Lorentz violating parameters in the fermionic sector, obtained from clock comparison experiments. (2) The calculation of radiative corrections in preferred frames associated to space granularity in the framework of a Yukawa model for the interactions and (3) The calculation of synchrotron radiation in the framework of the Myers-Pospelov effective theories describing Lorentz invariance violations, as well as a generalized approach to radiation in Planck scale modified electrodynamics. The above exploratory results show that quantum gravity phenomenology provides observational guidance in the construction of quantum gravity theories and opens up the possibility of probing Planck scale physics.

\section{Introduction}

Most theories of gravity suggest that our notion of space-time as a continuum needs to be revised at short distances (high energies) of the order of the Planck length $\ell_{P} \approx 10^{-33} \mathrm{~cm}$ (Planck mass, $M_{P} \approx 10^{19} \mathrm{GeV}$ ). At these scales quantum effects should be important and since space-time is to be considered as a set of dynamical interacting variables, instead of a mere background where physics occurs, the quantum nature of them together with their corresponding fluctuations could induce modifications to our standard notion of space-time. The consequences of space-time been considered as a continuum have been already successfully probed up to the much lower energies of $\approx 10^{3}$ $\mathrm{GeV}$, corresponding to the standard model of particle physics. The sixteen orders of magnitude between our current experimental arena and the region 
where such new effects would become relevant provides in fact plenty of room to look for modifications of our generally accepted ideas of space-time. We will refer to this new possibility as space-time presenting a granular or foamy structure in the following. Central to this question is the long time honored problem of finding a consistent unification of gravity and quantum mechanics, which nowadays is been actively pursued by loop quantum gravity [1] and string theory [2], among others lines of research. At any rate, a complete formulation of quantum gravity must explain how the standard notion of space-time at macroscopical length scales is recovered, thus validating the many successful test of classical Einstein gravity that have been performed and which have served to make sure that the correct starting point to construct the full theory has been taken.

Then a reasonable question to ask is whether or not such short length (high energy) effects leave any imprint in the dynamics of particles at standard model energies, which we could be able to detect with present day technology and observational sensibilities.

On one hand, from a purely phenomenological point of view one would expect some modifications to arise in the same way as particle propagation properties change when they move in a medium, with respect to those in the vacuum. Of course the analogy of a modified structure of space-time with a propagating medium is at most very tentative because the idea of a medium presupposes the existence of something external in which it is embedded. On the contrary, space-time is the arena where phenomena occur and there is nothing external to it. Nevertheless it is very plausible that a drastic change in our description of space-time would induce modifications in the way we deal with physics at such very short scales. Here we take the point of view that there is a remnant of such modifications at standard model energies, which are described by an effective field theory valid up to scales much lower than the Planck mass. These corrections manifest themselves as additional terms contributing to the propagation and interactions of known particles.

On the other hand, for a long time it has been taken for granted that Planck scale phenomena is completely out of reach from present experiments and/or observations (astrophysical, for example). Recent investigations show that this is definitely not the case, thus opening the door to a new area of research called Quantum Gravity Phenomenology (QGP) that is designed to use existing and forthcoming experiments and/or observations to restrict or constraint competing theories of quantum gravity based on their predicted imprints at these lower energies [3. A partial list of references describing such efforts is given in Ref. 4, 5]. This is definitely a great advance over the purely aesthetically criteria that prevailed before. Even though QGP has made independent advances in restricting the different parameters encoding some of the proposed modifications induced at standard model energies, there still remain the open problem of calculating them as a rigorous semiclassical approximation of a fundamental theory. Only after this gap is filled one could 
really be in position to use the observations to restrict the latter. The work presented here summarizes an heuristical step towards the estimation of the flat space semiclassical limit in the realm of LQG, together with further elaborations upon the results obtained in this way. Also some closely related topics originating from different approaches are discussed.

Before going to the details, let us briefly describe some of the alternative points of view to be found in the literature regarding the question of whether or not a granular or foamy structure of space would induce modifications to particle dynamics at standard model energies. Such modifications have been mainly understood in terms of the violation of standard Lorentz covariance, either through the introduction of preferred reference frames or by means of the inclusion of an extended or deformed Lorentz relativity. In the following we will generically refer to theses possibility as Lorentz invariance violation (LIV).

A first viewpoint is that modifications that manifest themselves in the form of LIV, do not arise. In this way covariance under the standard Lorentz transformations would be perfectly compatible with a discrete nature of space at Planck scale, which is encoded in the discrete spectrum of area and volume operators in LGQ, for example. This would be analogous to the well known property that the discrete spectrum of the angular momentum operator does not preclude the invariance of a system under the continuous rotation group [6. 7].

A second possibility is that corrections in the form of LIV do in fact arise. Within this point of view different alternatives have been also considered, which can be separated in two further categories:

(i) The first one can be characterized by a phenomenological parameterization of all possible corrections terms, according to the dimensionality of the corresponding LIV operators, which are assumed to arise via a spontaneous Lorentz symmetry breaking of a more fundamental model like string theory, for example [8]. These vacuum expectation values define a set of preferred frames, called concordant frames [9], in which the LIV terms can be maintained appropriately small when going from one frame to another via a passive (observer) Lorentz transformation. This is analogous to the description of atomic phenomena in the presence of an external magnetic field, where rotational invariance is broken by active (particle) transformation. Nevertheless, one can rotate the apparatus and perform the experiment in the presence of the rotated external field. The Standard Model Extension (SME) 11] belongs to this class and it has been highly successful in allowing a unified description of the great amount of experimental data that has been gathered since 1960 [10] in relation to the experimental verification of the isotropy of apace, the transformation properties among inertial frames and the validity of the discrete transformations $\mathrm{C}, \mathrm{P}$ and $\mathrm{T}$ as well as its possible combinations 12]. The SME has been recently generalized to incorporate gravity [13. More recently LIV models based upon dimension five operators 
have been constructed [14] and thoroughly analyzed in Refs. 15] 16. Within the approach (i) we find also direct extensions of Dirac and Maxwell equations incorporating modifications which go beyond effective field theories [17. An alternative approach to LIV via spontaneous symmetry breaking can be found in Ref. 18.

(ii) A second broad category of researches which leads to dynamical corrections can be distinguished by an effort to obtain such modifications either from an extension or deformation of the standard Lorentz relativity principle 19, 20, 21, 22, 23, 24, or from a fundamental description of quantum gravity like, for example: an effective field theory description of quantum general relativity [25, 26, a version of non-critical string theory [27, 28, 29] or the approach of loop quantum gravity [30, 31, 32, 33, 34, 35. Alternative ways of incorporating such dynamical modifications can be found in Ref. 36 37.

The most immediate way in which the corrections to the dynamics show up is through modifications to the energy-momentum relations for the particles 38, 39

$$
\begin{aligned}
\omega_{ \pm}^{2} & =|\boldsymbol{k}|^{2} \pm \xi \frac{|\boldsymbol{k}|^{3}}{E_{Q G}} \\
E_{R, L}^{2} & =|\boldsymbol{p}|^{2}+m^{2}+\eta_{R, L} \frac{|\boldsymbol{p}|^{3}}{E_{Q G}},
\end{aligned}
$$

as shown above for photons and fermions, respectively. The connection of such modifications with gravity, together with the possible astrophysical observation of the energy-dependent velocity in high-energy photons arriving from cosmological sources was suggested in Ref. 40. In fact, the frequency dependent photon velocity

$$
\left|\boldsymbol{v}_{ \pm}\right|=\frac{\partial \omega}{\partial|\boldsymbol{k}|}=\left(1 \pm \xi \frac{3}{2} \frac{|\boldsymbol{k}|}{E_{Q G}}+\ldots\right)
$$

predicts a time delay $\Delta t$ in the arrival of two photons having an energy difference $\Delta E$ and travelling a distance $L$, given by

$$
\Delta t \approx \xi \frac{\Delta E}{E_{Q G}} \frac{L}{c} .
$$

Here $E_{Q G}$ denotes the scale where quantum gravity effects become relevant, which is usually taken as the Planck energy. As emphasized in Ref. 40, the tiny contribution of $\xi / E_{Q G}$ can be amplified by selecting large energy differences or, more effectively, distances $L$ of cosmological magnitude. It is important to emphasize that this time delay is a purely kinematical consequence of the modified dispersion relations. The orders of magnitude $L \approx 10^{10}$ l.y., $\Delta E \approx 20 \mathrm{MeV}, E_{Q G}=10^{19} \mathrm{GeV}, \xi \approx 1$, lead to $\Delta t \approx 10^{-3} \mathrm{~s}$, which is within the range of sensitivities $\delta t$ in actual and forthcoming gamma ray bursts (GRB) observations. In order to measure such effect it is necessary 
Table 1. Bounds upon the quantum gravity scale $E_{Q G} / \xi$

\begin{tabular}{lllll}
\hline \multicolumn{1}{c}{$z$} & $\delta t(s)$ & \multicolumn{1}{c}{ Objects } & \multicolumn{1}{c}{$E_{Q G} / \xi(\mathrm{GeV})$} & Source \\
\hline 0.031 & 280 & Markarian421 & $>4.0 \times 10^{16}$ & Ref. [4] \\
$0.0085-3.42$ & 0.064 & GRB's (BATSE) $_{16}$ & $>6.9 \times 10^{15}$ & Ref. [4] \\
0.3 & 0.031 & GRB021206 (RHESSI) & $>1.3 \times 10^{17}$ & Ref. [42] \\
\hline
\end{tabular}

that $\delta t<\Delta t$. Present sensitivities allow for estimates of the lower bounds for $E_{Q G} / \xi$ presented in Table 1 Future planned observations of GRB at cosmological distances having well determined red shifts $z$, together with greater sensitivities ranging form $10^{-6} s$ (RHESSI: Reuben Ramaty High Energy Solar Spectrometer) to $10^{-7} s$ (GLAST: Gamma Ray Large Area Telescope ) will allow a substantial increase of such bounds. The fireball model of GRB emission [43, 44] predicts also the generation of $10^{5}-10^{10} \mathrm{GeV}$ neutrino bursts which will be detected by observatories like NUBE (Neutrino Burst Experiment), OWL (Orbiting Wide-angle Light collector experiment) and EUSO (Extreme Universe Space Observatory), for example. This will open up the possibility of using such particles, some times detected in coincidence with the respective photons, to set further bounds upon the quantum gravity scale $E_{Q G}$.

Very soon after the proposal of Ref. [40, the first derivation of a consistent electrodynamics leading to the dispersion relations (1) was obtained in the framework of a loop quantum gravity inspired model [30]. The resulting modified Maxwell equations are

$$
\begin{gathered}
\nabla \cdot \boldsymbol{E}=0, \quad \partial_{t} \boldsymbol{E}=-\nabla \times \boldsymbol{B}+2 \xi \ell_{P} \nabla^{2} \boldsymbol{B}, \\
\nabla \cdot \boldsymbol{B}=0, \quad \partial_{t} \boldsymbol{B}=+\nabla \times \boldsymbol{E}-2 \xi \ell_{P} \nabla^{2} \boldsymbol{E},
\end{gathered}
$$

leading to the energy-momentum relation

$$
\omega_{ \pm}=|\boldsymbol{k}|\left(1 \mp 2 \xi \ell_{P}|\boldsymbol{k}|\right) .
$$

Additional bounds upon the parameters describing the quantum gravity induced modifications have been obtained by incorporating the dynamics through the SME or similar constructions. In particular, the topics of Lorentz and CPT violations have been thoroughly studied in low energy physics via theory and experiments related to: Penning traps, clock comparison measurements, hydrogen-antihydrogen studies, spin polarized dispersion and muon experiments, among others subjects [45].

Finally we mention the use of astrophysical phenomena to discuss such modified theories. Distinguished examples are the bounds imposed by polarization measurements from astrophysical sources [46], the study of ultra high energy physics processes, among them cosmic rays, 47, 48, 49, 50, 51. and the consequences of the detected synchrotron radiation from the Crab 
nebulae, as well as that from other objects [15, 16, 52, 53. For recent reviews about such topics see Ref. 54 .

This contribution summarizes the work carried over in collaborations with different colleagues and it is organized as follows. Section 2 contains a nonspecialist review of some basic elements of LQG to be subsequently employed in the estimation of the induced dynamical modifications. In section 3 we describe the general features involved in the heuristical calculation of the effective photon and fermion Hamiltonians incorporating Planck scale corrections induced by LQG. These Hamiltonians are presented in subsection 3.3 together with some related comments. The remaining Section 4 is devoted to selected phenomenological applications of models presenting Planck scale dynamical corrections. Using existing data from clock-comparison experiments, in subsection 4.1 we obtain stringent bounds upon combinations of parameters appearing in the effective fermionic Hamiltonian previously derived. The incorporation of radiative corrections to the description of LIV effects in preferred frames associated to space granularity is discussed in subsection 4.2, leading to severe fine-tuning and naturalness problems. Subsection 4.3 contains the discussion of synchrotron radiation in LIV electrodynamics, emphasizing the model of Ref. [14, which is phenomenologically formulated as a theory parameterizing LIV with dimension five operators. Finally, a unified description of radiation in Planck scale modified electrodynamics including different models is presented in subsection 4.4

\section{Basic elements from Loop Quantum Gravity (LQG)}

Detailed reviews of LQG can be found in Refs. [1, [55. Here we just indicate some of the basic features that will be relevant in our heuristic calculation and present an intuitive, non-specialist introduction to this approach. LQG turns out to be the formulation of Einstein gravity as a Hamiltonian gauge theory with additional constraints, written in terms of non-local gauge covariant, diffeomorphisms invariant quantities. It is formulated in four dimensions and the matter couplings are obtained by rewriting the standard ones in terms of the new variables. This background independent, non-perturbative theory has allowed substantial progress in the old problem of producing a consistent quantum description of Einstein gravity. It has successfully dealt with traditional problems like a macroscopic account of the black hole entropy [56] and the construction of non-singular cosmological models [57, among other topics. One of its most notable predictions is the property that area and volume operators are quantized in terms of the corresponding powers of $\ell_{P}$, thus signaling a granular structure of space at short distances [58. It is precisely the possible consequences that such granularity may induce at energy scales of the standard model what is to be explored in this work. This question is intimately related to the still open problem of the semiclassical limit in 
LQG: how does one recover the continuous metric description of space-time starting from the quantum version of it?.

\subsection{The passage to the new variables}

LQG is formulated in terms of the Ashtekar-Barbero variables that arise as a canonical transformation from the usual ADM variables. Here we summarize the main steps leading to this choice and closely follow Ref. [55].

Let us start from the standard Einstein-Hilbert action for gravity in the signature $(-,+,+,+)$

$$
S\left[g_{\mu \nu}\right]=\frac{1}{2 \kappa} \int\left(d^{4} x\right) \sqrt{-\operatorname{det}\left(g_{\mu \nu}\right)}{ }^{(4)} R, \quad \kappa=\frac{8 \pi G}{c^{3}},
$$

where ${ }^{(4)} R$ is the four dimensional Ricci scalar. The ADM variables result from a foliation of spacetime in 3D surfaces $\Sigma$ of constant parameter $t$, described by coordinates $x^{a}, a=1,2,3$, together with the parameterization of the ten variables $g_{\mu \nu}$ in terms of the six components on the induced threemetric $q_{a b}$ on $\Sigma$, plus the three components of the shift vector $N^{a}$ and the lapse function $N$. In terms of these variables the four dimensional metric is

$$
g_{t t}=q_{c d} N^{c} N^{d}-N^{2}, \quad g_{t a}=g_{a t}=q_{a c} N^{c}, \quad g_{a b}=q_{a b}
$$

and the action (8) reads

$S\left[q_{a b}, N^{a}, N\right]=\frac{1}{2 \kappa} \int d t \int\left(d^{3} x\right) \sqrt{\operatorname{det}\left(q_{a b}\right)} N\left({ }^{(3)} R+K_{a b} K^{a b}-\left(K_{a}^{a}\right)^{2}\right)$,

where all the indices are now lowered or raised by $q_{a b}$ and its inverse $q^{c d}$. Here ${ }^{(3)} R$ is the Ricci scalar of the manifold $\Sigma$. The information about the velocities $\dot{q}_{a b}$ is contained in the extrinsic curvature

$$
K_{a b}=\frac{1}{2 N}\left(\dot{q}_{a b}-\mathcal{L}_{N} q_{a b}\right)=K_{b a},
$$

where $\mathcal{L}_{N}$ denotes de Lie derivative along the vector $N^{a}$. Introducing the canonical momenta

$$
\begin{aligned}
& \frac{1}{\kappa} \Pi^{a b}=\frac{\delta S}{\delta \dot{q}_{a b}}=\frac{1}{\kappa}\left(K^{a b}-q^{a b} K_{c}^{c}\right) \sqrt{\operatorname{det}\left(q_{a b}\right)}, \\
& \Pi=\frac{\delta S}{\delta \dot{N}}=0, \quad \Pi_{a}=\frac{\delta S}{\delta \dot{N}^{a}}=0
\end{aligned}
$$

and making a Legendre transformation one finds

$$
S\left[q_{a b}, \Pi^{a b}, \quad N^{a}, N\right]=\frac{1}{2 \kappa} \int d t \int\left(d^{3} x\right)\left[\Pi^{a b} \dot{q}_{a b}-N^{a} H_{a}-N H\right] .
$$


The above action shows that gravity is a fully constrained theory with zero canonical Hamiltonian. The invariance under local diffeomorphisms in $\Sigma$ is generated by the constraints $H_{a}$, while the dynamics is generated by the Hamiltonian constraint $H$. These are first class constraints which can be written as explicit functions of the canonical variables. The shift and the lapse functions turn out to be Lagrange multipliers.

The symplectic structure corresponding to (14) is given by the Poisson brackets

$$
\left\{\Pi^{a b}(\boldsymbol{x}), q_{c d}(\boldsymbol{y})\right\}=2 \kappa \delta_{(c}^{a} \delta_{d)}^{b} \delta^{(3)}(\boldsymbol{x}, \boldsymbol{y}),
$$

with the remaining ones being zero.

The second step towards the construction of the new variables is to introduce a non-abelian $S O(3) \approx S U(2)$ formulation of the action (14). To this end one rewrites the three-metric in terms of a triad $e_{a}^{i}$ such that

$$
q_{a b}=e_{a}^{i} e_{b}^{j} \delta_{i j}
$$

where the indices $i, j=1,2,3$, transform under local rotations.

The canonical variables arising in this step are the densitisized (weight +1 ) triad

$$
E_{i}^{a}=\frac{1}{2} \epsilon^{a b c} \epsilon_{i j k} e_{b}^{j} e_{c}^{k}
$$

together with the projected extrinsic curvature

$$
K_{a}^{i}=K_{a b} E_{j}^{b} \delta^{i j} .
$$

In terms of them we can rewrite

$$
\operatorname{det}\left(q_{a b}\right)=\operatorname{det}\left(E_{i}^{a}\right), \quad \dot{q}_{a b} \Pi^{a b}=2 \dot{K}_{a}^{i} E_{i}^{a},
$$

and the action is

$$
\begin{array}{r}
S\left[E_{i}^{a}, K_{b}^{j}, N^{a}, N, N^{i}\right]=\frac{1}{\kappa} \int d t \int\left(d^{3} x\right)\left[E_{i}^{a} \dot{K}_{a}^{i}-N^{a} H_{a}(E, K)\right. \\
\left.-N H(E, K)-N^{i} G_{i}(E, K)\right],
\end{array}
$$

The introduction of an extra $S O(3)$ gauge freedom in (16) requires the additional constraints

$$
G_{i}(E, K)=\epsilon_{i j k} E^{a j} K_{a}^{k}
$$

which turn out to be just the non-abelian Gauss law.

The final step to the Ashtekar-Barbero variables [59] starts from the recognition that there is a natural $S O(3) \approx S U(2)$ connection $\Gamma_{a}^{i}$ compatible with the triad, such that

$$
\partial_{[a} e_{b]}^{i}+\epsilon_{j k}^{i} \Gamma_{[a}^{j} e_{b]}^{k}=0 .
$$

Moreover, the triad introduced in Eq.(16) can be expressed in terms of the densitized triad as 


$$
e_{a}^{i}=\frac{1}{2 \sqrt{|\operatorname{det}(E)|}} \epsilon_{a b c} \epsilon^{i j k} E_{j}^{b} E_{k}^{c}, \quad e_{i}^{a}=\frac{\operatorname{sgn}(\operatorname{det}(E))}{\sqrt{|\operatorname{det}(E)|}} E_{i}^{a} .
$$

The final Ashtekar-Barbero connection, which allows to write the constraints in a simpler form, is

$$
A_{a}^{i}=\Gamma_{a}^{i}+\gamma K_{a}^{i} .
$$

Here $\gamma$ is the Immirzi parameter [60 and the corresponding field strength two-form is

$$
F^{i}=d A^{i}+\epsilon_{j k}^{i} A^{j} \wedge A^{k}, \quad F_{a b}^{i j}=\epsilon_{k}^{i j} F_{a b}^{k} .
$$

The final action results

$$
\begin{array}{r}
S\left[E_{i}^{a}, A_{b}^{j}, N^{a}, N, N^{i}\right]=\frac{1}{\kappa} \int d t \int\left(d^{3} x\right)\left[E_{i}^{a} \dot{A}_{a}^{i}-N^{a} H_{a}(E, A)\right. \\
\left.-N H(E, A)-N^{i} G_{i}(E, A)\right],
\end{array}
$$

with the canonical variables satisfying the non-zero Poisson brackets

$$
\left\{E_{j}^{b}(\boldsymbol{y}), A_{a}^{i}(\boldsymbol{x})\right\}=\kappa \gamma \delta_{a}^{b} \delta_{j}^{i} \delta^{(3)}(\boldsymbol{x}, \boldsymbol{y}) .
$$

The explicit form of the constraints are

$$
\begin{aligned}
& G_{i}=D_{a} E_{i}^{a}, \quad H_{b}=E_{i}^{a} F_{a b}^{i}-\left(1+\gamma^{2}\right) K_{b}^{i} G_{i}, \\
& H=\frac{1}{\sqrt{|\operatorname{det}(E)|}}\left(F_{a b}^{i j}-2\left(1+\gamma^{2}\right) K_{[a}^{i} K_{b]}^{j}\right) E_{i}^{a} E_{j}^{b} .
\end{aligned}
$$

The choice $\gamma=i$ corresponds to the original Ashtekar variables, which define a complexified version of Einstein gravity together with a very simple structure for the Hamiltonian constraint. The price to be paid is the need to incorporate some reality conditions in order to recover the corresponding real formulation.

Following the standard Dirac quantization procedure arising from the action (26), together with the Poisson brackets (27) in the coordinate space defined by the connection $A_{a}^{i}$, we promote the canonical variables to operators in such a way that

$$
\hat{A}_{a}^{i} \Psi[A]=A_{a}^{i} \Psi[A], \quad \hat{E}_{i}^{a} \Psi[A]=-i \hbar \kappa \gamma \frac{\delta}{\delta A_{a}^{i}} \Psi[A]
$$

and impose the operator version of the constraints as null conditions upon the wave function.

\subsection{Holonomies and fluxes}

Next we describe the fundamental operator variables employed to formulate LQG and which supersede the quantum version of the previous canonical variables (17) and (24). The basic support for the operators in LQG are open 

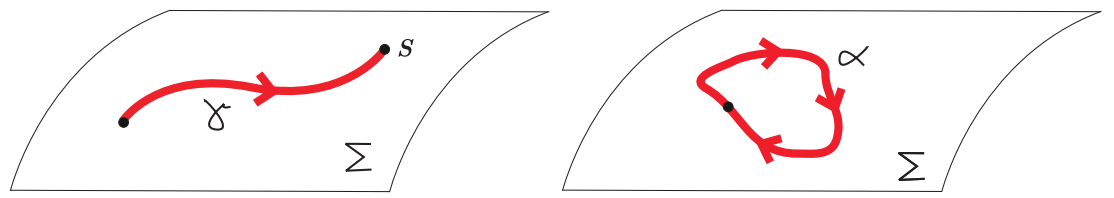

Fig. 1. Open and closed curves in $\Sigma$

or closed curves in $\Sigma$, as depicted in Fig. [1 respectively, together with twodimensional surfaces. These allow to define appropriately smeared versions of the operators (17) and (24). The $S U(2)$ connection operators $A_{a}^{i}(\boldsymbol{x})$ are replaced by holonomies (or parallel transport matrices) $U$ along open or closed curves in the thee-manifold $\Sigma$, defined as

$$
U(\gamma, A)=\mathcal{P} \exp i \int_{0, \gamma}^{s} A_{a}^{i}\left(\gamma\left(s^{\prime}\right)\right) \sigma_{i} \frac{d x^{a}}{d s^{\prime}} d s^{\prime} \in S U(2) .
$$

The notation is the following: the Pauli matrices $\sigma^{i}$ are the generators of the $S U(2)$ Lie algebra. The open curve $\gamma$ is parameterized by $s^{\prime}$ with $0<s^{\prime}<s$ and $\mathcal{P}$ denotes the standard path-ordered product. The holonomy, which is a multiplicative operator, transforms covariantly under $S U(2)$ gauge transformations at the end points, but still it is not invariant under diffeomorphisms.

The canonically conjugated momenta operators $\hat{E}_{i}^{a}$ are replaced by their corresponding fluxes over surfaces $S$ in $\Sigma$

$$
\hat{E}_{i}(S)=-i \hbar \int_{S} d \sigma^{1} d \sigma^{2} n_{a}(\boldsymbol{\sigma}) \frac{\delta}{\delta A_{a}^{i}(x(\boldsymbol{\sigma}))}, \quad n_{a}=\epsilon_{a b c} \frac{\partial x^{b}}{\partial \sigma^{1}} \frac{\partial x^{c}}{\partial \sigma^{2}} .
$$

Here $\boldsymbol{\sigma}=\left(\sigma^{1}, \sigma^{2}\right)$ are the intrinsic coordinates of the surface and $n_{a}$ is its normal vector. The above definitions (30) and (31) allow to calculate the corresponding commutator, which turns out to be a non-canonical one.

Quantum states $\Psi_{\Gamma, f}(A)$ are represented by functionals of generalized connections $(S U(2)$ group elements) defined over graphs in $\Sigma$, which are called cylindrical functions and define the kinematical space of the problem. As represented in Fig. [2] a graph $\Gamma=\left\{\boldsymbol{V}_{1}, \ldots, \boldsymbol{V}_{n} ; \gamma_{1}, \ldots, \gamma_{m}\right\}$ is a set of points $\left\{\boldsymbol{V}_{1}, \ldots, \boldsymbol{V}_{n}\right\} \in \Sigma$, called the vertices, joined by curves $\gamma_{1}, \ldots, \gamma_{m}$, called the edges of the graph. The number of edges attached to a vertex is called the valence of the vertex. To each edge of the graph we associate a group element $U(\gamma, A)$ labelled by an irreducible representation of $S U(2)$ and consider a function $f$ which is a map from the direct product of group representations to the complex numbers, so that

$$
\Psi_{\Gamma, f}(A)=f\left(U\left(\gamma_{1}, A\right), \ldots, U\left(\gamma_{m}, A\right)\right) \in \text { Kinematical Space. }
$$

For a given graph $\Gamma$ we define the scalar product of two associated functions $f$ and $g$ as 


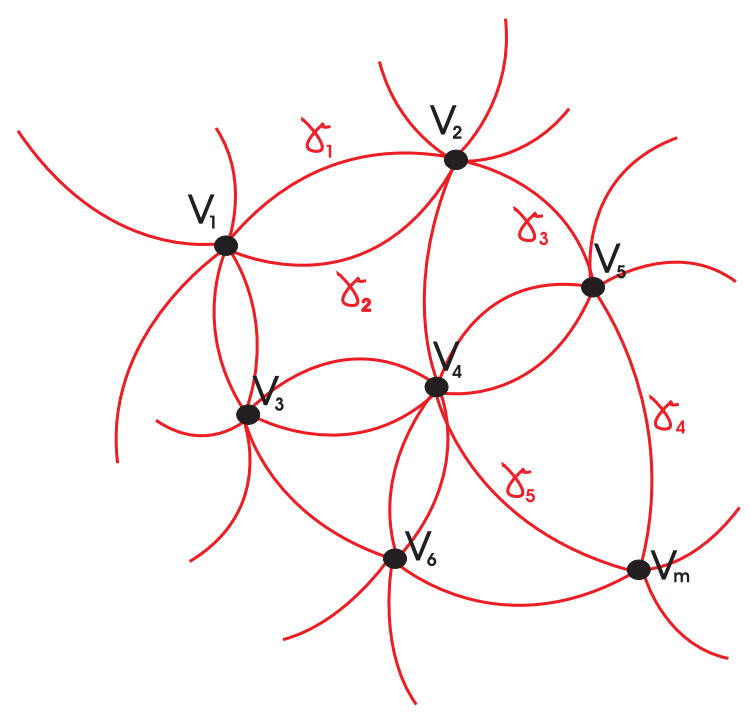

Fig. 2. Piece of a graph $\Gamma=\left\{\boldsymbol{V}_{1}, \ldots, \boldsymbol{V}_{n} ; \gamma_{1}, \ldots, \gamma_{m}\right\}$

$$
<\Psi_{\Gamma, f} \mid \Psi_{\Gamma, g}>=\int\left(\prod_{\gamma_{i}} d U_{i}\right) f^{*}\left(U_{1} \ldots U_{m}\right) g\left(U_{1} \ldots U_{m}\right),
$$

where we have denoted $U_{i}:=U\left(\gamma_{i}, A\right)$. Here $d U_{i}$ is the corresponding Haar measure for the group $S U(2)$.

There is still a long way to construct the physical space. To this end one has to subsequently impose $S U(2)$ gauge invariance, three dimensional diffeomorphism invariance and finally the Hamiltonian constraint. The first two conditions can be explicitly accomplished. The first step is implemented by an adequate choice of the functions $f$ in terms of the so-called intertwiners at each vertex. These are invariant tensors in $S U(2)$ which map the product of representations at each vertex into gauge invariant expressions. In these way the so called spin network states are defined and it is possible to prove that they form an orthonormal basis for the $S U(2)$ gauge invariant kinematical space. The second step is performed by going from loops to knots, thus defining equivalent classes of loops under diffeomorphisms.

The action of the operators (30) and (31) upon quantum states (33) is defined by

$$
\begin{aligned}
\left(U_{B}^{A}(A, \gamma) \Psi\right)[A] & =U_{B}^{A}(A, \gamma) \times \Psi[A] \\
\hat{E}_{i}(S) U(A, \gamma) & =\sum_{k} \pm i \hbar U\left(A, \gamma_{1}^{P_{k}}\right) \sigma_{i} U\left(A, \gamma_{2}^{P_{k}}\right)
\end{aligned}
$$

Thus, holonomies act as multiplicative operators, while the action of the smeared conjugated momentum depends upon a given edge $\gamma$ of the graph crossing or not the associated surface $S$ at the points $P_{k}$. For each intersection 
$P_{k}$ the path $\gamma$ is separated in two pieces $\gamma_{1}^{P_{k}}$ and $\gamma_{2}^{P_{k}}$ such that $\gamma=\gamma_{1}^{P_{k}} \circ \gamma_{2}^{P_{k}}$. The action at that intersection insert the generator $\sigma_{i}$ between the holonomies corresponding to each of these paths. This is shown schematically in Fig. (3). To close this summary we mention the area operator $\hat{A}$ which is defined in

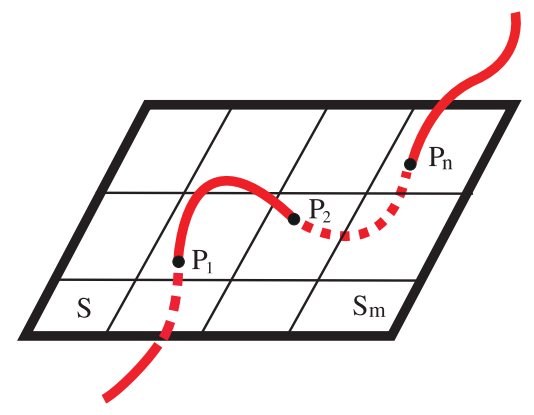

Fig. 3. Conditions for the action of the smeared momentum operator

the following way

$$
\hat{A}=\lim _{n \rightarrow \infty} \sum_{n} \sqrt{\hat{E}_{i}\left(S_{n}\right) \hat{E}_{i}\left(S_{n}\right)}
$$

by partitioning the associated surface $S$. A similar construction is made for the volume operator $\hat{V}$. These are well defined partial observables (hermitian operators in the $S U(2)$ gauge invariant kinematical space) with discrete eigenvalues and eigenfunctions given by the spin network states. An important property to be used in the following is that only the nodes contribute to the action of the volume operator upon states defined in a graph.

\subsection{Elements of Thiemann regularization}

Thiemann has proposed a general regularization scheme that produces a consistent mathematical definition for the operators entering in the description of loop quantum gravity 61, 62. Such regularized operators act upon states which are functions of generalized connections defined over graphs. The regularization procedure is based upon a triangulation of space which is adapted to each graph. This means that the space surrounding any vertex of $\Gamma$ is filled with tetrahedra $\Delta$ having only one vertex in common with the graph (called the basepoint $\boldsymbol{V}(\Delta)$ ) plus segments $\boldsymbol{U}_{I}(\Delta), I=1,2,3$, starting at $\boldsymbol{V}$ and directed along the edges of the graph. For a vertex of valence greater than three one must consider all possible combination of three edges to build the tetrahedra. In the regions not including the vertices of $\Gamma$ the choice of tetrahedra is arbitrary and the results are independent of it. The open path along the segment $\boldsymbol{U}_{I}$ is denoted by $\gamma_{\boldsymbol{V}, \boldsymbol{U}_{I}}$. The arc connecting the end points of $\boldsymbol{U}_{I}(\Delta)$ and $\boldsymbol{U}_{J}(\Delta)$ is denoted by $a_{I J}(\Delta)$ and the loop 
$\alpha_{\boldsymbol{V}, \boldsymbol{U}_{I}, \boldsymbol{U}_{J}}:=\gamma_{\boldsymbol{V}, \boldsymbol{U}_{I}} \circ a_{I J} \circ \gamma_{\boldsymbol{V}, \boldsymbol{U}_{J}}^{-1}:=\alpha_{I J}(\Delta)$ can be formed. This construction is illustrated in the basic tetrahedron depicted in Fig. (4).

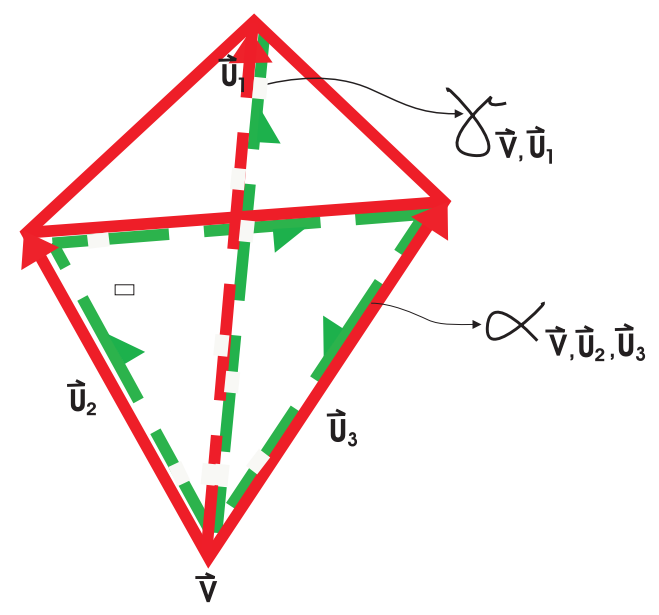

Fig. 4. Basic tetrahedron and paths in Thiemann regularization

A first step in the implementation of the regularization method is to express each connection and field strength in terms of an adequate holonomy. For example, using the path $\gamma_{\boldsymbol{V}, \boldsymbol{U}_{1}}$, of coordinate length $\epsilon$, we can incorporate a connection $A_{a}(\boldsymbol{x})$ into the corresponding holonomy via the expansion

$$
U\left(\gamma_{\boldsymbol{V}, \boldsymbol{U}_{\mathbf{1}}}, A\right)=1+\epsilon U_{1}^{a} A_{a}(x)+O\left(\epsilon^{2}\right)
$$

An analogous calculation for the closed triangular path $\alpha_{\boldsymbol{V}, \boldsymbol{U}_{2}, \boldsymbol{U}_{3}}$ leads to the following expression incorporating the curvature

$$
U\left(\alpha_{\boldsymbol{V}, \boldsymbol{U}_{2}, \boldsymbol{U}_{3}}, A\right)=1+\frac{1}{2} \epsilon^{2} U_{2}^{a} U_{3}^{b} F_{a b}+O\left(\epsilon^{3}\right) .
$$

A second ingredient of the method is the basic identity 61

$$
\frac{E_{i}^{a}(\boldsymbol{x}) E_{j}^{b}(\boldsymbol{x}) \epsilon^{i j k}}{\sqrt{\operatorname{det}(E(\boldsymbol{x}))}}=\frac{2}{\kappa} \epsilon^{a b c}\left\{A_{c}^{k}, V(R)\right\}_{P B}, \quad V=\int_{R}\left(d^{3} x\right) \sqrt{\operatorname{det}\left(E^{a i}\right)},
$$

where $R$ is a region such $\boldsymbol{x} \in R$. In this way the volume $V$, which will be promoted to the operator level once the quantization is performed, is introduced in the regularization.

To fix ideas let us look at the first piece of the smeared Hamiltonian constraint

$$
\left.\mathcal{H}_{1}=\int\left(d^{3} x\right) N(x) \frac{F_{a b}^{k} \epsilon^{i j}{ }_{k} E_{i}^{a} E_{j}^{b}}{\sqrt{\operatorname{det}(E)}}=\int\left(d^{3} x\right) N(x) \epsilon^{a b c} F_{a b}^{i}\left\{A_{c i}, V\right\}\right),
$$


where we have used Eq.(38) in the second step. Now, substituting the expressions (36) and (37) for $A_{a}, F_{a b} \in S U(2)$, respectively, and replacing the integral with a sum, we can write

$$
\begin{aligned}
\mathcal{H}_{1}=\lim _{\epsilon \rightarrow 0} \sum_{m} \epsilon^{3} N_{m} \epsilon^{I J K} \frac{1}{\epsilon^{3}} \operatorname{Tr}\left(\left(U\left(\alpha_{\boldsymbol{V}_{m}, \boldsymbol{U}_{I}, \boldsymbol{U}_{J}}, A\right)-U^{-1}\left(\alpha_{\boldsymbol{V}_{m}, \boldsymbol{U}_{I}, \boldsymbol{U}_{J}}, A\right)\right)\right. \\
\left.\times U^{-1}\left(\gamma_{\boldsymbol{V}_{m}, \boldsymbol{U}_{K}}, A\right)\left\{U\left(\gamma_{\boldsymbol{V}_{m}, \boldsymbol{U}_{K}}, A\right), V\left(R_{m}\right)\right\}\right) .
\end{aligned}
$$

The crucial fact is that the divergent contribution arising from $1 / \sqrt{\operatorname{det}(E)}$, which goes like $1 / \epsilon^{3}$, is cancelled by the correspondent factors contained in expansion of the holonomies. In this way Eq. (40) leads to a well defined regulated expression. Furthermore, when the correspondent quantities are quantized, the volume operator $\hat{V}$ is naturally introduced. This has the important consequence that the action of such operators upon wave functions defined on a graph get contributions only from the nodes of the graph.

Another illustrative example of this regularization is the magnetic sector of the electromagnetic Hamiltonian

$$
H_{\text {Maxwell }}^{B}=\int_{\Sigma} d^{3} x \frac{q_{a b}}{2 Q^{2} \sqrt{\operatorname{det} q}} \underline{B}^{a} \underline{B}^{b},
$$

which is translated into the operator expression

$$
\begin{aligned}
\hat{H}^{B}= & \frac{1}{2 \ell_{P}^{4} Q^{2}} \sum_{\boldsymbol{V} \in V(\Gamma)} \sum_{\boldsymbol{V}(\Delta)=\boldsymbol{V}\left(\Delta^{\prime}\right)=\boldsymbol{V}} \epsilon^{J K L} \epsilon^{M N P} \times \\
& \times \hat{w}_{i L \Delta} \hat{w}_{i P \Delta^{\prime}}\left(\underline{h}_{\alpha_{J K}(\Delta)}-1\right)\left(\underline{h}_{\alpha_{M N}\left(\Delta^{\prime}\right)}-1\right),
\end{aligned}
$$

where

$$
\hat{w}_{k I \Delta}=\operatorname{Tr}\left(\tau_{k} h_{\boldsymbol{U}_{I}(\Delta)}\left[h_{\boldsymbol{U}_{I}(\Delta)}^{-1}, \sqrt{\hat{V}_{v}}\right]\right) .
$$

Here we have simplified the notation by introducing

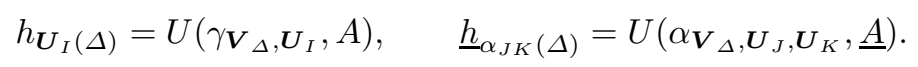

The underlined quantities (holonomy and connection) refer to the electromagnetic sector, and are to be distinguished from the gravitational ones.

In the following we will separately consider each matter contribution to the Hamiltonian constraint as the corresponding Hamiltonian for the respective gravitationally coupled sector.

\section{A kinematical estimation of the semiclassical limit}

The construction of states that live in the physical space of LQG and which approximate a given geometry at large distances, while retaining their granular structure at Planck length scales is still an open problem. This is usually referred to as the semiclassical limit of LQG and it is actively under investigation [63]. 


\subsection{Heuristic characterization of the states}

In order to make some preliminary steps towards the study of the consequences of space granularity at large scales in the flat space limit, we take here an heuristical point of view, starting from the exact and well understood operator version of LQG. The approximation we introduce consists in defining their action upon the semiclassical states through some plausible requirements, without having an explicit construction for such states. We think of the semiclassical configuration corresponding to a particular matter or gauge field, described by operators collectively denoted by $\hat{F}(\boldsymbol{x})$, plus gravity as given by an ensemble of graphs $\Gamma$, each occurring with probability $P(\Gamma)$. To each of such graphs we associate a wave function $|\Gamma, \mathcal{L}, F\rangle:=|\Gamma, S\rangle$ which we assume to be peaked with respect to the classical matter field configuration $F(\boldsymbol{x})$, together with a flat gravitational metric and a zero value for the gravitational connection at large distances. Not surprisingly, the semiclassical approximation gives us simultaneous average information regarding a field together with its canonically conjugated momentum. In other words, the contribution of the gravitational and matter operators inside the expectation value is estimated as 31, 32, 33.

$$
\begin{aligned}
& \left\langle\Gamma, S\left|\ldots \hat{q}_{a b} \ldots\right| \Gamma, S\right\rangle \approx \delta_{a b}+O\left(\ell_{P} / \mathcal{L}\right) \\
& \left\langle\Gamma, S\left|\ldots \hat{A}_{i a} \ldots\right| \Gamma, S\right\rangle \approx\left(0+(1 / \mathcal{L})\left(\ell_{P} / \mathcal{L}\right)^{\gamma}\right)_{i a}, \\
& \left\langle\Gamma, S\left|\ldots \hat{\hat{F}}_{a b} \ldots\right| \Gamma, S\right\rangle \approx \mu \underline{F}_{a b}
\end{aligned}
$$

and similarly for any product of operators inside the expectation value. As an illustration, the gauge field is taken to be the electromagnetic field in the following but an analogous estimation will hold for any other gauge or matter fields. The parameter $\Upsilon \geq 0$ is a real number and $\underline{F}_{a b}$ denotes the classical electromagnetic field strength. We further associate the effective hamiltonian

$$
H_{\Gamma}=\left\langle\Gamma, S\left|\hat{H}_{\Gamma}\right| \Gamma, S\right\rangle
$$

to each graph.

The coarse graining scale $\mathcal{L}>>\ell_{P}$ of the wave function is such that a continuous flat metric approximation is appropriate for distances $D$ much larger that $\mathcal{L}$, while the granular structure of space becomes relevant when probing distances $D$ smaller that $\mathcal{L}$. That is to say, $\mathcal{L}$ is not a scale of quantum gravity but rather it is a scale that separates the continuum description of space from its discrete characterization that is fully manifest at the Planck length $\ell_{P}$, which signals the quantum behavior. Summarizing, we expect the following behavior

$$
\begin{aligned}
D & >\mathcal{L}>>\ell_{P}: \text { continuous flat classical geometry, } \\
\ell_{P} & \sim D<<\mathcal{L}: \text { manifest discrete structure of space, }
\end{aligned}
$$


for the probe scale $D$. The coarse graining scale $\mathcal{L}$ is not provided by our approximation and has to be estimated in each particular case. We will explore some alternatives in subsection 3.4

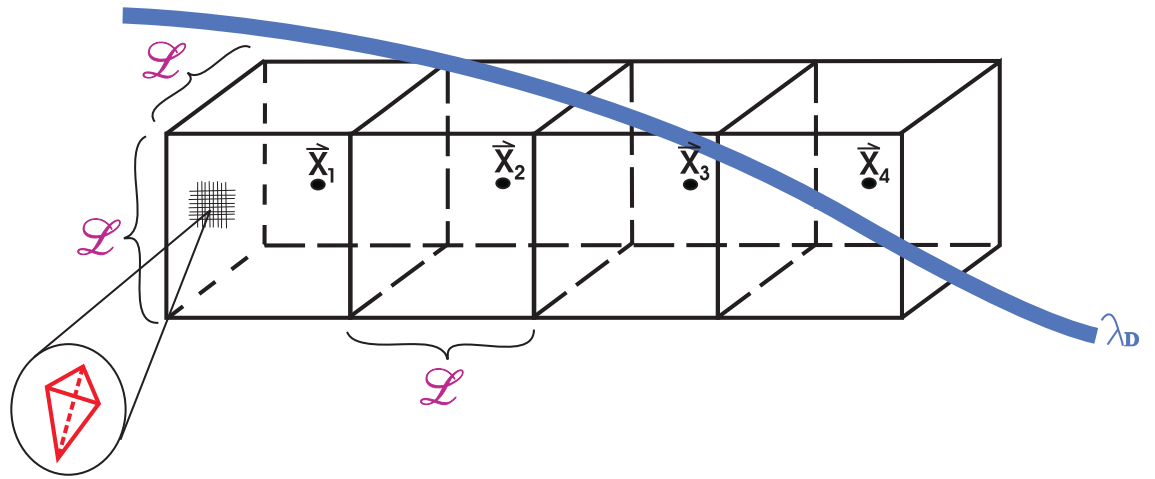

Fig. 5. Different scales for the model of space considered in the text

As indicated in Fig. 5 we think of space as constructed by adding boxes of size $\mathcal{L}^{3}$, which center represents a given point $\mathbf{x}$ in the continuum limit and which contain a large number of vertices of the adapted graphs. The matter field $F(\boldsymbol{x})$, characterized by a de Broglie wave length $\lambda$, is considered as a slowly varying function within each box $(\lambda>\mathcal{L})$ and contributes with its classical value at the center of the box, when taking expectation values. The requirement $\lambda>\mathcal{L}$ guarantees that we can describe the flat space dynamics in terms of an effective field theory, using the standard differential calculus of the continuum. On the other hand, gravitational variables are rapidly varying inside each box.

The total effective Hamiltonian is defined as an average over the graphs $\Gamma$

$$
\mathrm{H}=\sum_{\Gamma} P(\Gamma) \mathrm{H}_{\Gamma}
$$

\subsection{The calculation}

We summarize now the method of calculation [31, 32, 33. For each graph $\Gamma$ the effective Hamiltonian is defined by (46). For a given vertex $\boldsymbol{V}$ inside the expectation value, one expands each holonomy or flux operator in powers of the segments $\left.U_{I}^{a}(\Delta)\right)$ of the attached tetrahedra $\Delta$, plus derivatives of the gauge or matter fields operators. Schematically, in the case of (42) this produces

$$
H_{\Gamma}^{B}=\sum_{\boldsymbol{V} \in \boldsymbol{V}(\Gamma)} \sum_{\boldsymbol{V}(\Delta)=\boldsymbol{V}}\langle\Gamma, S|\left(\partial^{a_{1}} \partial^{a_{2}} \ldots \partial^{a_{k}} \ldots \hat{\hat{F}}_{p_{1} q_{1}}(\boldsymbol{V})\right) \ldots \times
$$




$$
\times\left(\ldots \partial^{a_{m-1}} \partial^{a_{m}} \underline{\hat{F}}_{p q}(\boldsymbol{V})\right) \hat{T}_{a_{1} \ldots a_{m}}^{p_{1} q_{1} \ldots p q}\left(\boldsymbol{V}, \boldsymbol{U}_{I}(\Delta), \hat{A}_{i a}, \hat{V}\right)|\Gamma, S\rangle .
$$

Here $\hat{T}$ contains gravitational operators (connection and volume operators, for example) together with contributions depending on the segments of the adapted triangulation in the particular graph. Next, according to Fig. 5 space is considered to be divided into boxes, each centered at a fixed point $\boldsymbol{x}$ and with volume $\mathcal{L}^{3} \approx d^{3} x$. The choice of boxes is the same for all the graphs considered. Each box contains a large number of vertices of the semiclassical state $\left(\mathcal{L} \gg \ell_{P}\right)$, but it is considered as infinitesimal in the scale where the space can be regarded as continuous. The sum over the vertices in (48) is subsequently split as a sum over the vertices in each box, plus a sum over the boxes. Also, one assumes that the gauge or matter operators are slowly varying within a box $(\mathcal{L}<<\lambda$, with $\lambda$ been the correspondent particle wavelength), in such a way that for all the vertices inside a given box one can write $\left\langle\Gamma, S\left|\ldots \underline{\hat{F}}_{a b}(\boldsymbol{V}) \ldots\right| \Gamma, S\right\rangle=\mu \underline{F}_{a b}(\boldsymbol{x})$, for example. Here $\underline{F}_{a b}$ is the classical electromagnetic field at the center of the box and $\mu$ is a dimensionless constant which is determined in such a way that the standard classical result in the zeroth order approximation is recovered. Applying the procedure just described to (48) leads to

$$
\begin{aligned}
H_{\Gamma}^{B}= & \sum_{\operatorname{Box}}\left(\partial^{a_{1}} \partial^{a_{2}} \ldots \partial^{a_{k}} \ldots \underline{F}_{p_{1} q_{1}}(\boldsymbol{x})\right) \ldots\left(\ldots \partial^{a_{m-1}} \partial^{a_{m}} \underline{F}_{p q}(\boldsymbol{x})\right) \times \\
& \sum_{\boldsymbol{V} \in \operatorname{Box}} \ell_{P}^{3} \sum_{\boldsymbol{V}(\Delta)=\boldsymbol{V}} \mu^{n+1}\left\langle\Gamma, S\left|\frac{1}{\ell_{P}^{3}} \hat{T}_{a_{1} \ldots a_{m}}{ }^{n q \ldots p_{1} q_{1}}\left(\boldsymbol{V}, \boldsymbol{U}(\Delta), A_{i a}\right)\right| \Gamma, S\right\rangle,
\end{aligned}
$$

where $n+1$ is the total number of factors $F_{r s}(\boldsymbol{x})$, each of which can contain some derivatives. The expectation value of the gravitational contribution is supposed to be a rapidly varying function inside each box. Next we consider the total effective Hamiltonian (47), which is defined as an average over the graphs $\Gamma$, i.e. over the adapted triangulations. This effectively amounts to average the remaining expectation values in each box of the sum (49). We call this box-average $t_{a_{1} \ldots a_{m}} p q \ldots p_{1} q_{1}(\boldsymbol{x})$ and define it by

$$
\begin{array}{r}
\sum_{\Gamma} P(\Gamma) \sum_{\boldsymbol{V} \in \operatorname{Box}} \ell_{P}^{3} \sum_{\boldsymbol{V}(\Delta)=\boldsymbol{V}}\left\langle\Gamma, S\left|\frac{1}{\ell_{P}^{3}} \hat{T}_{a_{1} \ldots a_{m}}{ }^{p q \ldots p_{1} q_{1}}\right| \Gamma, S\right\rangle= \\
d^{3} x t_{a_{1} \ldots a_{m}} p q \ldots p_{1} q_{1}(\boldsymbol{x}) .
\end{array}
$$

We estimate $t_{a_{1} \ldots a_{m}} p q \ldots p_{1} q_{1}(\boldsymbol{x})$ by demanding it to be constructed from the flat space tensors $\delta_{a b}$ and $\epsilon_{a b c}$, together with the corresponding ones $\tau_{i}$ and $\delta_{i}^{a}$, in the case of spinors. In this way we are imposing isotropy and rotational invariance on our final Hamiltonian in the frame selected by the choice of the semiclassical states. This is somewhat analogous to the spontaneously 
symmetry breaking scheme, where the choice of the vacuum selects a particular frame for the symmetry breaking vacuum expectation values. Also, the scalings given in (45) together with the additional assumptions

$$
\langle\Gamma, S|\ldots \hat{V} \ldots| \Gamma, S\rangle \longrightarrow \ell_{P}^{3}, \quad U_{I}^{a} \longrightarrow \ell_{P},
$$

are used in the above estimation. After replacing the summation over boxes by the integral over space, the resulting Hamiltonian has the final form

$$
\begin{aligned}
H^{B}=\int d^{3} x\left(\partial^{a_{1}} \partial^{a_{2}} \ldots \partial^{a_{k}} \ldots \underline{F}_{p_{1} q_{1}}(\boldsymbol{x})\right) \ldots\left(\ldots \partial^{a_{m-1}} \partial^{a_{m}} \underline{F}_{p q}(\boldsymbol{x})\right) \\
\times t_{a_{1} \ldots a_{m}}{ }^{p q \ldots p_{1} q_{1}}(\boldsymbol{x}) .
\end{aligned}
$$

Finally we exhibit some of the required expansions. The basic electromagnetic holonomy around the path $\alpha_{J K}$

$$
\underline{h}_{\alpha_{J K}(\Delta)}=e^{-i \int_{\alpha_{J K}(\Delta)} d t \dot{U}^{a}(t) \underline{\hat{A}}_{a}(s(t))}=e^{-i \Phi^{B}\left(F_{J K}\right)},
$$

can be written in terms of the magnetic flux $\Phi^{B}$ through the face bounded by such closed path. The expansion of such flux with respect to the magnetic field at the vertex is

$$
\begin{aligned}
\Phi^{B}\left(F_{I J}\right)=( & +\frac{1}{3}\left(U_{I}^{c}+U_{J}^{c}\right) \partial_{c}+\frac{1}{12}\left(U_{I}^{c} U_{I}^{d}+U_{I}^{c} U_{J}^{d}+U_{J}^{c} U_{J}^{d}\right) \\
& \left.\times \partial_{c} \partial_{d}+\ldots\right) \frac{1}{2} U_{I}^{a} U_{J}^{b} \epsilon_{a b c} B^{c}(\boldsymbol{V}),
\end{aligned}
$$

to second order in the derivatives.

The expansion of the gravitational operator (43) is

$$
\hat{w}_{i L \Delta}=U_{L}^{a} w_{i a}+U_{L}^{a} U_{L}^{b} w_{i a b}+U_{L}^{a} U_{L}^{b} U_{L}^{c} w_{i a b c}+\mathcal{O}\left(s^{4} w\right),
$$

to third order in the vectors $U_{L}^{a}$. The remaining operators in the expansion include the connection together with the volume operators, in the form

$$
\begin{aligned}
w_{i a}= & \frac{1}{2}\left[A_{i a}, \sqrt{V}\right], \quad w_{i a b}=\frac{1}{8} \epsilon_{i j k}\left[A_{j a},\left[A_{k b}, \sqrt{V}\right]\right] \\
& w_{i a b c}=-\frac{1}{48}\left[A_{j a},\left[A_{j b},\left[A_{i c}, \sqrt{V}\right]\right]\right] .
\end{aligned}
$$

\subsection{The results}

The application of the method described in the previous section leads to the following effective Hamiltonians. In the electromagnetic case we obtain 32 


$$
\begin{aligned}
H^{E M}= & \frac{1}{Q^{2}} \int d^{3} \boldsymbol{x}\left[\left(1+\theta_{7}\left(\frac{\ell_{P}}{\mathcal{L}}\right)^{2+2 \Upsilon}\right) \frac{1}{2}\left(\underline{\boldsymbol{B}}^{2}+\underline{\boldsymbol{E}}^{2}\right)\right. \\
& +\theta_{8} \ell_{P}(\underline{\boldsymbol{B}} \cdot(\boldsymbol{\nabla} \times \underline{\boldsymbol{B}})+\underline{\boldsymbol{E}} \cdot(\boldsymbol{\nabla} \times \underline{\boldsymbol{E}})) \\
& +\theta_{3} \ell_{P}^{2}\left(\underline{B}^{a} \nabla^{2} \underline{B}_{a}+\underline{E}^{a} \nabla^{2} \underline{E}_{a}\right)+\theta_{2} \ell_{P}^{2} \underline{E}^{a} \partial_{a} \partial_{b} \underline{E}^{b} \\
& \left.+\theta_{4} \ell_{P}^{2} \mathcal{L}^{2}\left(\frac{\ell_{P}}{\mathcal{L}}\right)^{2 \Upsilon}\left(\underline{\boldsymbol{B}}^{2}\right)^{2}+\ldots\right] .
\end{aligned}
$$

The above result has been extended to the Yang-Mills case in Ref. 64. The case where only $\theta_{8}$ in non zero corresponds to the Gambini-Pullin effective Hamiltonian [30].

In the case of Majorana fermions with mass $m$ we have [31, 33]

$$
\begin{array}{r}
H^{F}=\int d^{3} x\left[i \pi ( \boldsymbol { x } ) \left(1+\kappa_{1}\left(\frac{\ell_{P}}{\mathcal{L}}\right)^{1+\Upsilon}+\kappa_{2}\left(\frac{\ell_{P}}{\mathcal{L}}\right)^{2+2 \Upsilon}+\right.\right. \\
\left.+\frac{\kappa_{3}}{2} \ell_{P}^{2} \nabla^{2}\right) \tau^{d} \partial_{d} \xi(\boldsymbol{x}) \\
+\frac{i}{4} \frac{1}{\mathcal{L}} \pi(\boldsymbol{x})\left(\kappa_{4}\left(\frac{\ell_{P}}{\mathcal{L}}\right)^{\Upsilon}+\kappa_{5}\left(\frac{\ell_{P}}{\mathcal{L}}\right)^{1+2 \Upsilon}+\ldots+\frac{\kappa_{7}}{2} \ell_{P}^{2} \nabla^{2}\right) \xi(\boldsymbol{x}) \\
\left.+\frac{m}{2} \xi^{T}(\boldsymbol{x})\left(i \sigma^{2}\right)\left(1+\kappa_{8}\left(\frac{\ell_{P}}{\mathcal{L}}\right)^{1+\Upsilon}+\left(\kappa_{9} \ell_{P}+\ldots\right) \tau^{a} \partial_{a}\right) \xi(\boldsymbol{x})+\text { c.c. }\right],
\end{array}
$$

where $\pi(\boldsymbol{x})=i \xi^{*}(\boldsymbol{x})$ is the canonically conjugated momentum of the twocomponent spin $1 / 2$ field $\xi(\boldsymbol{x})$. The notation is $\tau^{a}=-(i / 2) \sigma^{a}$, where $\sigma^{a}$ are the standard Pauli matrices.

Some comments regarding the procedure are now in order. (i) The dimensionless numerical coefficients $\theta_{A}, \kappa_{A}$ appearing in the above effective Hamiltonians remain arbitrary in the procedure, but are independent of any parameter of the model. To predict them will require an exact knowledge of the states used to calculate the corresponding average values, together with the action of the basic operators upon them. In this respect we have obtained a systematic parameterization of the possible modified Hamiltonians in terms of higher derivative operators, where the dependence upon the scales of the problem $\left(\ell_{P}, \mathcal{L}, \Upsilon\right)$ has been explicitly determined. In particular, these coefficients could turn out to be zero, leading to no dynamical corrections. (ii) A main drawback of the method is that it does not incorporate properly the dynamics via the Hamiltonian constraint of quantum gravity. An improved semiclassical approximation taking care of the many issues assumed or left over in our approach is certainly needed. (iii) There is also the question of interpreting the results (56) and (57) in relation to their transformation properties under standard active (particle)global Lorentz transformations. There 
are various possibilities. These Hamiltonians have been calculated in a specific reference frame where isotropy under rotations is maintained. This could signal the presence of an absolute reference frame, a reborn version of the ether. This hypothesis will be further explored in the following and has received a lot of attention in the framework of different models in the literature. On the other hand one could argue that this specific frame has been selected by the choice of the semiclassical states that describe the flat space limit, in analogous way to the spontaneously symmetry breaking procedure. The transformation properties under this assumption have not been studied. The third alternative is that only a complete calculation of the corrections to flat space dynamics will tell us which is the proper generalization, if any, of the global Lorentz transformations, thus yielding a deformed or extended version for preserving the equivalence of inertial frames and leading to a modified relativity principle. (iv) Finally we must say that the above results have provided some motivation for phenomenological theories which have explored the consequences of such modifications and have shown that either experimental or astrophysical observations, even with the actual level of sensitivity, can set rather stringent bounds upon the correction parameters. In other words, Planck scale sensitivity has been already attained in a rather broad set of observations. Conversely, this would imply severe constraints upon the fundamental theory once the semiclassical limit is correctly performed.

\subsection{The parameters $\mathcal{L}$ and $\Upsilon$}

In order to produce numerical estimations for some of the effects arising from the previously obtained modifications to flat space dynamics, we must further fix the value of the scales $\mathcal{L}$ and $\Upsilon$.

Let us recall that $\mathcal{L}$ is a scale indicating the onset of the distance from where the non perturbative states in the loop representation can be approximated by the classical flat metric. The propagating particle is characterized by energies which probe distances of the order of the De Broglie wave length $\lambda$. As mentioned previously, just to be consistent with a description in terms of classical continuous equations it is necessary to require that $\mathcal{L}<\lambda$. Two distinguished cases for choosing $\mathcal{L}$ arise: (i) the mobile scale, where we take the marginal choice $\mathcal{L}=\lambda$ in each situation and (ii) the universal scale, which has been introduced in the discussion of the GZK anomaly [50]. The consideration of the different reactions involved produces the preferred range $4.6 \times 10^{-8} \mathrm{GeV}^{-1} \geq \mathcal{L} \geq 8.3 \times 10^{-9} \mathrm{GeV}^{-1}$. A recent study of the gravitational Cerenkov effect together with neutrino oscillations 65] yields a universal scale evaluation which is consistent with the former.

Ranges for $\Upsilon$ have been estimated considering the observation that atmospheric neutrino oscillations at average energies of the order $10^{-2}-10^{2} \mathrm{GeV}$ are dominated by the corresponding mass differences via the oscillation length $L_{m}$ [66. This means that additional contributions to the oscillation length, in particular the quantum gravity correction $L_{Q G}$, should satisfy $L_{Q G}>L_{m}$. 
This has been used to set lower bounds upon $\Upsilon$. Within the proposed two methods for estimating the scale $\mathcal{L}$ of the process we obtain: (i) $\Upsilon>0.15$ when $\mathcal{L}$ is considered as a mobile scale and (ii) $\Upsilon>1.2$ when the scale $\mathcal{L}$ takes the universal value $\mathcal{L} \approx 10^{-8} \mathrm{GeV}^{-1} 33$. These results are summarized in Table 2

Table 2. Ranges for the parameters $\mathcal{L}$ and $\Upsilon$

\begin{tabular}{lcc}
\hline & $\mathcal{L}\left[\mathrm{GeV}^{-1}\right]$ & $\Upsilon$ \\
\hline Mobile scale & $\mathcal{L}=\lambda$ & $\Upsilon>0.15$ \\
Universal scale & $8.3 \times 10^{-9}<\mathcal{L}<4.6 \times 10^{-8}$ & $\Upsilon>1.2$ \\
\hline
\end{tabular}

\section{Phenomenological aspects}

In this section we discuss four phenomenological applications of models presenting Planck scale dynamical corrections. In subsection 4.1 we summarize the derivation of stringent bounds upon combinations of some parameters appearing in the effective fermionic Hamiltonian (57), using existing data from clock-comparison experiments. Subsection 4.2 is devoted to the calculation of radiative corrections arising in LIV effects with preferred frames associated to space granularity. In order to make the calculation simple we consider a Yukawa model for the interactions. Subsection 4.3 contains the discussion of synchrotron radiation in Planck scale modified electrodynamics, emphasizing the model of Ref. 14] which is phenomenologically formulated as a theory parameterizing LIV with dimension five operators. Finally, a unified description of radiation in Planck scale modified electrodynamics incorporating different models is presented in subsection 4.4.

\subsection{Bounds on the fermionic sector parameters from clock-comparison experiments}

The Hamiltonian (57) for two components fermions was obtained under the assumption of flat space isotropy and it was assumed to account for the dynamics in a preferred reference frame, identified as the one in which the Cosmic Microwave Background looks isotropic. The earth velocity $\mathbf{w}$ with respect to that frame has already been determined to be $w / c \approx 1.23 \times 10^{-3}$ by COBE [67]. Thus, in the earth reference frame one expects the appearance of signals indicating minute violations of space isotropy encoded in the diurnal variation of the $\mathbf{w}$-dependent terms appearing in the transformed Hamiltonian or Lagrangian 68. On the other hand, many high precision experimental test of such variations, using atomic and nuclear systems for 
example, have been already reported in the literature [10, 69, 70, 71] and already analyzed in terms of the SME. Amazingly enough such precision is already adequate to set very stringent bounds on some of the parameters arising from the quantum gravity corrections.

We have considered the case of non-relativistic Dirac particles and obtained corrections which involve the coupling of the spin to the CMB velocity, together with a quadrupolar anisotropy of the inertial mass [68. The calculation was made with the choices $\Upsilon=0$ and $\mathcal{L}=1 / M$, where $M$ is the rest mass of the fermion. Here it is important to emphasize again that $\mathcal{L}>>\ell_{P}$ is not the scale of quantum gravity but rather the lower limit of distances from which a description of space as a continuum is already valid. In this sense we are taking the upper limit settled by the de Broglie wave length $\lambda_{D}=1 / M$ of the nucleon, which allows us to make sense of the corresponding Dirac equation. As we will show later, a lower limit for $\mathcal{L}$ will only make the bounds upon the LIV parameters more stringent.

Keeping only terms linear in $\ell_{P}$, the equations of motion for a Majorana fermion of mass $m$ described by the two-component spinor $\xi$, arising from the Hamiltonian (57) are given by

$$
\begin{aligned}
& {[i \partial / \partial t-i A \boldsymbol{\sigma} \cdot \boldsymbol{\nabla}+K / 2] \xi-m(\alpha-i \beta \boldsymbol{\sigma} \cdot \boldsymbol{\nabla}) \chi=0,} \\
& {[i \partial / \partial t+i A \boldsymbol{\sigma} \cdot \boldsymbol{\nabla}-K / 2] \chi-m(\alpha-i \beta \boldsymbol{\sigma} \cdot \boldsymbol{\nabla}) \xi=0,}
\end{aligned}
$$

where

$$
\begin{aligned}
& A=\left(1+\Theta_{1} m \ell_{p}\right), \quad \alpha=\left(1+\Theta_{3} m \ell_{P}\right), \\
& K=m \Theta_{4} m \ell_{P}, \quad \beta=\Theta_{2} \ell_{P} .
\end{aligned}
$$

The notation in Eqs. (58) is $\chi=-i \sigma_{2} \xi^{*}$ and it is a direct matter to verify the consistency between them. These equations can be readily extended to the Dirac case by considering $\chi$ and $\xi$ as independent spinors unified in $\Psi^{T}=\left(\xi^{T}, \chi^{T}\right)$, with the result

$$
\left(i \gamma^{\mu} \partial_{\mu}+\Theta_{1} m \ell_{P} i \gamma \cdot \nabla-\frac{K}{2} \gamma_{5} \gamma^{0}-m\left(\alpha-i \Theta_{2} \ell_{P} \Sigma \cdot \nabla\right)\right) \Psi=0 .
$$

Here we have used the representation in which $\gamma_{5}$ is diagonal and the spin operator is $\Sigma^{k}=(i / 2) \epsilon_{k l m} \gamma^{l} \gamma^{m}$, with standard particle physicist conventions. The normalization has been chosen so that in the limit $\left(m \ell_{P}\right) \rightarrow 0$ we recover the standard massive Dirac equation. The term $m\left(1+\Theta_{3} m \ell_{P}\right)$ can be interpreted as a renormalization of the mass whose physical value is taken to be $M=m\left(1+\Theta_{3} m \ell_{P}\right)$. After these modifications we can write an effective Lagrangian describing the time evolution as seen in the CMB frame. In order to obtain the dynamics in the laboratory frame we implement an observer (passive) Lorentz transformation in the former Lagrangian and rewrite it in a covariant looking form by introducing explicitly the CMB frame four velocity $W^{\mu}=\gamma(1, \mathbf{w} / c)$ arising from the boosted rest frame velocity $(1, \mathbf{0})$. The result is 


$$
\begin{aligned}
L_{D}= & \frac{1}{2} i \bar{\Psi} \gamma^{\mu} \partial_{\mu} \Psi-\frac{1}{2} M \bar{\Psi} \Psi+\frac{1}{2} i\left(\Theta_{1} M \ell_{P}\right) \bar{\Psi} \gamma_{\mu}\left(g^{\mu \nu}-W^{\mu} W^{\nu}\right) \partial_{\nu} \Psi \\
& +\frac{1}{4}\left(\Theta_{2} M \ell_{P}\right) \bar{\Psi} \epsilon_{\mu \nu \alpha \beta} W^{\mu} \gamma^{\nu} \gamma^{\alpha} \partial^{\beta} \Psi-\frac{1}{4}\left(\Theta_{4} M \ell_{P}\right) M W_{\mu} \bar{\Psi} \gamma_{5} \gamma^{\mu} \Psi+\text { h.c. }
\end{aligned}
$$

where $W^{\mu}$ is an external non-dynamical field which is not to be varied in the corresponding action. It is interesting to remark that the above Lagrangian provides a specific realization of the general form considered in the SME. According to Ref. 72, the identifications are

$$
\begin{aligned}
& a_{\mu}=H_{\mu \nu}=d_{\mu \nu}=e_{\mu}=f_{\mu}=0, \quad c_{\mu \nu}=\Theta_{1} M \ell_{P}\left(g_{\mu \nu}-W_{\mu} W_{\nu}\right), \\
& g_{\alpha \beta \gamma}=-\Theta_{2} M \ell_{P} W^{\rho} \epsilon_{\rho \alpha \beta \gamma}, \quad b_{\mu}=\frac{1}{2} \Theta_{4} M^{2} \ell_{P} W_{\mu} .
\end{aligned}
$$

The above reference provides also the non-relativistic limit of the Hamiltonian corresponding to the general SME modified fermion Lagrangian. In our case, up to first order in $\ell_{P}$ and up to order $(\mathbf{w}) / c^{2}$, the identifications (62) yield

$$
\begin{array}{r}
\tilde{H}=M c^{2}\left(1+\Theta_{1} M \ell_{P}(\mathbf{w} / c)^{2}\right)+\Theta_{1} M \ell_{P}\left[\frac{\mathbf{w} \cdot Q_{P} \cdot \mathbf{w}}{M c^{2}}\right] \\
+\left(1+2 \Theta_{1} M \ell_{P}\left(1+\frac{5}{6}(\mathbf{w} / c)^{2}\right)\right)\left(\frac{p^{2}}{2 M}+g \mu \mathbf{s} \cdot \mathbf{B}\right) \\
+\left(\Theta_{2}+\frac{1}{2} \Theta_{4}\right) M \ell_{P}\left[\left(2 M c^{2}-\frac{2 p^{2}}{3 M}\right) \mathbf{s} \cdot \frac{\mathbf{w}}{c}+\frac{1}{M} \mathbf{s} \cdot Q_{P} \cdot \frac{\mathbf{w}}{c}\right],
\end{array}
$$

where $s^{i}=\sigma^{i} / 2$.

The above effective Hamiltonian has been employed in the description of the valence nucleon responsible for the transitions measured in clockcomparison experiments using pairs of nuclei like $\left({ }^{21} \mathrm{Ne},{ }^{3} \mathrm{He}\right)$ 70, and $\left({ }^{129} \mathrm{Xe},{ }^{3} \mathrm{He}\right)$ [1], for example. In (63) we have not written the terms linear in the momentum since they average to zero in the nuclear bound state situation. Here $g$ is the standard gyromagnetic factor, and $Q_{P}$ is the momentum quadrupole tensor with components $Q_{P i j}=p_{i} p_{j}-1 / 3 p^{2} \delta_{i j}$. The terms in the second square bracket of the LHS of (63) represent a coupling of the spin to the velocity of the privileged reference frame. The first term inside the bracket has been measured with high accuracy and an upper bound for the coefficient has been found. The second term in the same bracket is a small anisotropy contribution and can be neglected. Thus we find the correction

$$
\delta H_{S}=\left(\Theta_{2}+\frac{1}{2} \Theta_{4}\right) M \ell_{P}\left(2 M c^{2}\right)\left[1+O\left(\frac{p^{2}}{2 M^{2} c^{2}}\right)\right] \mathbf{s} \cdot \frac{\mathbf{w}}{c} .
$$

The first square bracket in the LHS of (63) represents an anisotropy of the inertial mass and has been bounded in Hughes-Drever like experiments. With the approximation $Q_{P}=-5 / 3<p^{2}>Q / R^{2}$ for the momentum quadrupole 
moment, with $Q$ being the electric quadrupole moment and $R$ the nuclear radius, we obtain

$$
\delta H_{Q}=-\Theta_{1} M \ell_{P} \frac{5}{3}\left\langle\frac{p^{2}}{2 M}\right\rangle\left(\frac{Q}{R^{2}}\right)\left(\frac{w}{c}\right)^{2} P_{2}(\cos \theta),
$$

for the quadrupole mass perturbation, where $\theta$ is the angle between the quantization axis and $\mathbf{w}$. Using $<p^{2} / 2 M>\sim 40 \mathrm{MeV}$ for the energy of a nucleon in the outer shell of a typical heavy nucleus, together with the experimental bounds of references 70,71 we find 68

$$
\left|\Theta_{2}+\frac{1}{2} \Theta_{4}\right|<2 \times 10^{-9}, \quad\left|\Theta_{1}\right|<3 \times 10^{-5} .
$$

From Eqs. 64) and 65) we realize that only products of the type (LIV parameters) $\times 1 / \mathcal{L} \approx$ (LIVparameters) $\times M$ are bounded by the experiments. In this way choosing a smaller $\mathcal{L}$, i.e. a larger $M$, will just decrease the upper bound for the LIV parameters. Thus the choice $\mathcal{L}=\lambda_{D}=1 / M$ produces the weakest bound in this analysis.

\subsection{Radiative corrections in preferred frames modelling space granularity}

So far corrections to the dynamics have arisen only in the non-interacting theory, through factors of the type $\left(E / M_{P}\right)^{\gamma}$, which are directly relevant at unaccessible energies $E \approx M_{P}$. A possibility of probing such high energies is through the inclusion of radiative corrections (particle's self energies, for example ) because the internal momenta are integrated up to the maximum allowed in a given reference frame. The standard folklore with respect to any new physics entering at high scales (here Planck scale) is that it has negligible effects on the leading-order low-energy physics (here free particle corrections). Contrary to this belief, we show in this subsection that modelling space granularity via the introduction of a physical cutoff, which defines a preferred reference frame, leads to unsuppressed dimension four LIV contributions 74, 75]. Results consistent with these have been obtained in Ref. 14.

To this end we consider the calculation of one-loop radiative corrections in the Yukawa model

$$
L=1 / 2(\partial \phi)^{2}-1 / 2 \mu^{2} \phi^{2}+\bar{\psi}\left(i \gamma^{\mu} \partial_{\mu}-m\right) \psi+g \phi \bar{\psi} \psi+(\mathrm{LVT}) .
$$

Here LVT refers to the highly suppressed zeroth order Lorentz violating terms that take into account the previously discussed free particle dynamical modifications. We model the space granularity by introducing a physical cut-off $\Lambda$ in such a way that the magnitude of the three-momentum in any loop is bounded by this quantity. The parameter $\Lambda$ defines the onset of the scale 
at which the granularity of space becomes manifest. A convenient way of incorporating this requirement is to introduce the physical cutoff function

$$
f(|\boldsymbol{k}| / \Lambda), \quad f(0)=1, \quad f(\infty)=0, \quad \Lambda \approx 1 / \mathcal{L},
$$

which suppresses the internal momenta having $|\boldsymbol{k}| \geq \Lambda$. One can visualize this function as a smoothed theta function with the correspondent jump at $|\boldsymbol{k}| \approx \Lambda$. Our choice is that the cutoff function depends on spatial momentum, but not of energy, and that it is rotationally invariant.

\section{The fermion self energy [75]}

Tests of Lorentz invariance typically deal with the relation between energy and 3-momentum of an isolated particle, which is given by the position of the pole of the particle's full propagator. We will calculate the effect of loop corrections on this relation. It is convenient, as usual, to write the full fermion propagator as

$$
S(p)=\frac{i}{p^{\mu} \gamma_{\mu}-m-\Sigma_{2}(p)},
$$

where $\Sigma_{2}(p)$ is the standard fermion self energy. Here we are neglecting the zeroth order corrections to the dispersion relations for the free particles.

As a first manner to model the validity of a continuous description of space only up to some short distance $1 / \Lambda \approx \mathcal{L}$, we choose to cutoff only the free scalar propagator, but not the free fermion propagator, which is sufficient to cutoff the UV divergence of the one-loop fermion self-energy while giving a maximally simple calculation. In this way, the one-loop approximation to the fermion self-energy is

$$
\Sigma_{2}(p)=i g^{2} \int \frac{\mathrm{d}^{4} k}{(2 \pi)^{4}} \frac{f(|\boldsymbol{k}| / \Lambda)}{k^{2}+i \epsilon} \frac{\gamma_{\rho}(p+k)^{\rho}+m}{(p+k)^{2}-m^{2}+i \epsilon},
$$

where we have set $\mu=0$ for simplicity. We wish to investigate the self-energy when the momentum $p^{\mu}$ and the mass $m$ are much less than the parameter $\Lambda$. If the integral were convergent as the parameter is removed $(\Lambda \rightarrow \infty)$, then the self-energy would equal its value at $\Lambda=\infty$ plus corrections of order $m / \Lambda$ and $p / \Lambda$. However, it is easy to see that the integral is divergent in this limit. To analyze its behavior, we observe that differentiating it twice with respect to $p$ gives a finite integral (when $\Lambda \rightarrow \infty$ ). So we extract the zeroth order and the linear terms in the momentum expansion of $\Sigma_{2}$ about $p=0$

$$
\Sigma_{2}(p)=\Sigma_{2}(p=0)+\left.p^{0} \partial_{p^{0}} \Sigma_{2}(p)\right|_{p=0}+\left.p^{i} \partial_{p^{i}} \Sigma_{2}(p)\right|_{p=0}+\hat{\Sigma}(p) .
$$

Now $\hat{\Sigma}(p)$ is $O\left(p^{2}\right)$ as $p \rightarrow 0$ and it is finite when the cutoff is removed. Therefore it only gives power-suppressed Lorentz violations. The unsuppressed Lorentz violations, if any, can only arise from the first three terms. We find that the terms $\Sigma_{2}(p=0),\left.\partial_{p^{0}} \Sigma_{2}(p)\right|_{p=0}$ and $\left.\partial_{p^{i}} \Sigma_{2}(p)\right|_{p=0}$ are proportional 
to the Dirac matrices $1, \gamma_{i}, \gamma^{0}$, exactly as in the Lorentz-invariant case; this follows from the discrete symmetries of our model, rather than full Lorentz invariance. Hence we can write

$$
\begin{aligned}
\Sigma_{2}(p) & =A m+p^{0} \gamma_{0} B+p^{i} \gamma_{i} C+\hat{\Sigma}(p, \Lambda=\infty)+O(p / \Lambda, m / \Lambda) \\
& =A m+p^{\mu} \gamma_{\mu} C+p^{0} \gamma_{0}(B-C)+\hat{\Sigma}(p, \Lambda=\infty)+O(p / \Lambda, m / \Lambda),
\end{aligned}
$$

where $A, B$ and $C$ are numerical-valued functions of the parameters of the problem. The only leading-power Lorentz violation is caused by the difference between the $B$ and $C$, which we calculate below and find it to be nonzero and unsuppressed.

In fact, the constant term is

$$
\Sigma_{2}(0)=\frac{i g^{2}}{(2 \pi)^{4}} \int \mathrm{d}^{4} k f(|\boldsymbol{k}| / \Lambda) \frac{\gamma_{\rho} k^{\rho}+m}{\left(k^{2}-m^{2}+i \epsilon\right)\left(k^{2}+i \epsilon\right)} .
$$

Since the physical cutoff depends only on $|\boldsymbol{k}|$, the modified theory is invariant under reversal of any component of $k^{\mu}$ and the term proportional to $\gamma_{\rho}$ vanishes after integration. Hence

$$
\begin{aligned}
A & =\frac{i g^{2}}{(2 \pi)^{4}} \int \mathrm{d}^{4} k \frac{f(|\boldsymbol{k}| / \Lambda)}{\left(k^{2}-m^{2}+i \epsilon\right)\left(k^{2}+i \epsilon\right)} . \\
& =\frac{g^{2}}{4 \pi^{2}} \int_{0}^{\infty} \mathrm{d} l f(l / \Lambda) \frac{l^{2}}{m^{2}}\left(\frac{1}{l}-\frac{1}{E_{l}}\right),
\end{aligned}
$$

where $E_{l}=\sqrt{l^{2}+m^{2}}$. In the second line, we have performed the integrals over $k^{0}$ and the angle of $\boldsymbol{k}$. The corresponding term in $\Sigma_{2}$ is proportional to the unit Dirac matrix, so that it is equivalent to a fermion mass term. Note that when $\Lambda \rightarrow \infty$ there is a logarithmic divergence, which can be removed by the usual mass renormalization. Since the term is proportional to the unit Dirac matrix, it does not violate Lorentz invariance.

The situation for the derivative terms is different. For the time-like derivative we have

$$
\left.\partial_{p^{0}} \Sigma_{2}(p)\right|_{p=0}=\frac{i g^{2}}{(2 \pi)^{4}} \int \mathrm{d}^{4} k \frac{f(|\boldsymbol{k}| / \Lambda)}{k^{2}+i \epsilon}\left[\frac{\gamma_{0}}{k^{2}-m^{2}+i \epsilon}-\frac{2 k^{0}\left(\gamma_{\rho} k^{\rho}+m\right)}{\left(k^{2}-m^{2}+i \epsilon\right)^{2}}\right] .
$$

Invariance under reversal of components removes all but the term proportional to $\gamma_{0}$, so that

$$
\begin{aligned}
B & =\frac{i g^{2}}{(2 \pi)^{4}} \int \mathrm{d}^{4} k \frac{f(|\boldsymbol{k}| / \Lambda)}{k^{2}+i \epsilon}\left[\frac{1}{k^{2}-m^{2}+i \epsilon}-\frac{2 k^{0^{2}}}{\left(k^{2}-m^{2}+i \epsilon\right)^{2}}\right] \\
& =\frac{g^{2}}{4 \pi^{2}} \int_{0}^{\infty} \mathrm{d} l f(l / \Lambda)\left[\frac{2 l^{2}\left(E_{l}-l\right)}{m^{4}}-\frac{l}{m^{2}}\right] .
\end{aligned}
$$


Similarly, from the space-like derivative we get

$$
\begin{aligned}
C & =\frac{i g^{2}}{(2 \pi)^{4}} \int \mathrm{d}^{4} k \frac{f(|\boldsymbol{k}| / \Lambda)}{k^{2}+i \epsilon}\left[\frac{1}{k^{2}-m^{2}+i \epsilon}+\frac{2 \boldsymbol{k}^{2} / 3}{\left(k^{2}-m^{2}+i \epsilon\right)^{2}}\right] \\
& =\frac{g^{2}}{4 \pi^{2}} \int_{0}^{\infty} \mathrm{d} l f(l / \Lambda)\left[\frac{2 l^{3}}{3 m^{4}}-\frac{l}{m^{2}}+\frac{l^{2}}{m^{2} E_{l}}-\frac{2 l^{4}}{3 m^{4} E_{l}}-\frac{l^{4}}{3 m^{2} E_{l}^{3}}\right]
\end{aligned}
$$

As expected, both $B$ and $C$ have logarithmic divergences as $\Lambda \rightarrow \infty$.

We are interested in the difference, which gives the low-energy violation of Lorentz invariance

$$
\begin{aligned}
B-C & =-\frac{2 i g^{2}}{(2 \pi)^{4}} \int \mathrm{d}^{4} k \frac{f(|\boldsymbol{k}| / \Lambda)}{k^{2}+i \epsilon} \frac{\frac{1}{3} \boldsymbol{k}^{2}+k^{0^{2}}}{\left(k^{2}-m^{2}+i \epsilon\right)^{2}} \\
& =\frac{g^{2}}{12 \pi^{2}} \int_{0}^{\infty} \mathrm{d} l f(l / \Lambda)\left[\frac{8 E_{l}^{3}}{m^{4}}-\frac{8 l^{3}}{m^{4}}-\frac{12 E_{l}}{m^{2}}+\frac{3}{E_{l}}+\frac{m^{2}}{E_{l}^{3}}\right] .
\end{aligned}
$$

Suppose the integral (78) were convergent in the continuum limit. Then it would be zero in this limit, as can be seen by Wick rotation of an integral of $\frac{1}{3} \boldsymbol{k}^{2}+k^{0^{2}}$ times an arbitrary Lorentz-invariant function $F\left(k^{2}\right)$. This argument depends on the integral being absolutely convergent, i.e., on the integral of the absolute value of the integrand being finite. In contrast, the corresponding integral for $B-C$ is logarithmically divergent. So the leading term for the integral, obtained when $\Lambda \rightarrow \infty$, depends on how this cutoff is removed. In our case this is dictated by the physical modelling of the space granularity via the function (68), as expressed in Eq.(79). This choice implies that the factor in square brackets behaves like $m^{2} / l^{3}$ when $l / m \rightarrow \infty$, so that the integral is finite when the cutoff is removed. Besides, we can write this square bracket as indicated in the second line of the following equation, which leads to a direct calculation of the leading term

$$
\begin{aligned}
\lim _{\Lambda \rightarrow \infty}(B-C) & =\frac{g^{2}}{12 \pi^{2}} \int_{0}^{\infty} \mathrm{d} l\left[\frac{8 E_{l}^{3}}{m^{4}}-\frac{8 l^{3}}{m^{4}}-\frac{12 E_{l}}{m^{2}}+\frac{3}{E_{l}}+\frac{m^{2}}{E_{l}^{3}}\right] \\
& =\frac{g^{2}}{12 \pi^{2} m^{4}} \int_{0}^{\infty} \mathrm{d} l \frac{\mathrm{d}}{\mathrm{d} l}\left[2 l E_{l}^{3}-2 l^{4}-3 m^{2} l E_{l}+\frac{m^{4} l}{E_{l}}\right] \\
& =\frac{g^{2}}{48 \pi^{2}} .
\end{aligned}
$$

The nonzero value of $B-C$ shows that the one-loop fermion self-energy introduces a Lorentz-violating term at small momenta even when the cut-off is made very large: Lorentz violation is suppressed not by a power of $m / \Lambda$, but only by a factor of the coupling. A convenient interpretation of this result uses the language of effective field theories. Observe that the Lorentz violation caused by the $B-C$ term is equivalent at the one-loop level to adding a term

$$
-i \xi \bar{\psi} \gamma_{\mu} W^{\mu} W^{\nu} \partial_{\nu} \psi
$$


to the Lagrangian. That is, we could obtain the same results in the $\Lambda \gg m$ region by dropping the $B-C$ term in the self-energy (72) and then adding this extra term to the Lagrangian. In this extra term $\xi=g^{2} / 48 \pi^{2}$, is the coefficient calculated above, and $W^{\mu}=(1,0)$ covariantly specifies the rest frame of the cutoff. Summarizing, the fermion self-energy contribution has the effect of feeding back terms of large unnatural size into the Lagrangian, thus producing a strong fine tuning problem when higher corrections are taken into account.

The boson self energy [7]

Similar results can be obtained from the boson self energy. Since the internal lines are both fermionic in this case, a more symmetrical way of incorporating the physical cutoff is required. This is accomplished by extending the fermionic propagators to

$$
\frac{i}{\gamma k-m+i \epsilon} \longrightarrow \frac{i f(|\boldsymbol{k}| / \Lambda)}{\gamma k-m+\Xi(\boldsymbol{k})+i \epsilon} .
$$

The function $\Xi(\boldsymbol{k})$ takes into account the modified dispersion relations of the theory to zeroth order and its contribution will not be taken into account in the following.

Consider only the physical momentum cutoff function and calculate the boson self-energy $\Pi(E, \boldsymbol{p}, \Lambda)$, defined in terms of the full propagator $\Delta(E, \boldsymbol{p})$

$$
\Delta(E, \boldsymbol{p})=\frac{-i f(|\boldsymbol{k}| / \Lambda)}{p^{2}-\mu^{2}-\Pi(E, \boldsymbol{p}, \Lambda)-i \epsilon}
$$

The one loop calculation is

$$
\begin{aligned}
\Pi(E, \boldsymbol{p}, \Lambda)= & -\frac{i g^{2}}{16 \pi^{4}} \int d^{4} k f(|\boldsymbol{k}| / \Lambda) f(|\boldsymbol{k}+\boldsymbol{p}| / \Lambda) \\
& \times \frac{\operatorname{Tr}[(\gamma \cdot k+m)(\gamma \cdot(k+p)+m)]}{\left(k^{2}-m^{2}+i \epsilon\right)\left[(k+p)^{2}-m^{2}+i \epsilon\right]} .
\end{aligned}
$$

The result can be presented as an expansion in even powers of the momenta

$$
\Pi(E, \boldsymbol{p}, \Lambda)=A+p^{2} B+\eta_{R C} p^{\mu} p^{\nu} W_{\mu} W_{\nu}+\hat{\Pi}(E, \boldsymbol{p}, \Lambda),
$$

where $\hat{\Pi}$ is convergent when the regulator is removed so that it contributes only with Lorentz violating terms which are suppressed by powers of $p^{2} / \Lambda^{2}$. In the frame where the cutoff $\Lambda$ is defined, $W_{\mu}=(1, \mathbf{0})$, we obtain

$$
\eta_{R C}=\frac{1}{2}\left(\left(\frac{\partial^{2} \Pi(p)}{\partial p^{0} \partial p^{0}}\right)_{p=0}+\left(\frac{\partial^{2} \Pi(p)}{\partial p^{1} \partial p^{1}}\right)_{p=0}\right),
$$

from (85). The calculation of the required pieces starts from (84) and after some algebra produces 


$$
\begin{aligned}
\left(\frac{\partial^{2} \Pi(p)}{\partial p^{0} \partial p^{0}}\right)_{p=0}=-\frac{g^{2}}{32 \pi^{2}} \int_{0}^{\infty} d k k^{4} \frac{f^{2}}{E_{k}^{5}}, \\
\left(\frac{\partial^{2} \Pi(p)}{\partial p^{i} \partial p^{j}}\right)_{p=0}=-\delta^{i j} \frac{g^{2}}{48 \pi^{2}}\left[\int _ { 0 } ^ { \infty } k ^ { 2 } d k \left(f f^{\prime \prime} \frac{k^{2}}{\Lambda^{2} E_{k}^{3}}+f f^{\prime} \frac{k}{\Lambda E_{k}^{5}}\left[k^{2}+4 m^{2}\right]\right.\right. \\
\left.\left.-f^{2} \frac{1}{2 E_{k}^{7}}\left[3 k^{4}+10 m^{2} k^{2}-3 m^{4}\right]\right)\right] .
\end{aligned}
$$

Here $f=f(k / \Lambda)$ and the derivatives, denoted by primes, are with respect to the argument of $f$. As usual we have $E_{k}=\sqrt{k^{2}+m^{2}}$. Each expression (87) and (88) is logarithmically divergent, but their combination (86) is finite. Thus we can estimate the leading contribution to $\eta_{R C}$ by setting $f=1$ inside the integrals with no derivatives with respect to $f$. The terms containing derivatives are handled by assuming that the contributions of $f^{\prime}, f^{\prime \prime}$ are sharply localized in the region $x=k / \Lambda \approx 1$. This implies that we neglect the mass contributions $m^{2}<<k^{2} \approx \Lambda^{2}$ in the corresponding integrals. Also, we set equal to zero the resulting boundary terms which include $f^{\prime}$ in the required integrations by parts. The final result is

$$
\eta_{R C}=\frac{g^{2}}{12 \pi^{2}}\left[1+2 \int_{0}^{\infty} d x x f^{\prime}(x)^{2}\right],
$$

which is estimated in the range $\eta_{R C} \geq 10^{-3}$ using standard model couplings. On the other hand, $\eta_{R C}$ can be interpreted as a correction $\delta c$ to the boson (photon) speed, for which extremely tight bounds exists

$$
\frac{\eta_{R C}}{2}=\frac{\delta c}{c}=\leq 10^{-20}
$$

The two examples presented here show that radiative corrections in preferred frames associated to space granularity, modelled by the physical cutoff function (68), induce LIV contributions which are enormously increased with respect to the already established bounds. That is to say, they are not suppressed by factors $1 / M$ as expected initially. In this way, a naturalness problem arises and a fine tuning problem appears when considering higher order radiative corrections. One possibility to guarantee the stability of dimension three and four LIV contributions is via the introduction of a custodial symmetry. This option has been explored using supersymmetry in Ref. [76].

An alternative way of dealing with radiative corrections in LIV processes can be found in Ref. 177 .

\subsection{Radiation in Lorentz violating electrodynamics}

The observation of $100 \mathrm{MeV}$ synchrotron radiation from the Crab Nebula has recently been used to impose stringent limits upon the parameters describing a modified electrodynamics embodied in Maxwell equations, which 
together with the corresponding coupled equations for the charges, get correction terms which are linear in the Planck length [15. Such bounds were based on a set of very reasonable assumptions on how some of the standard results of synchrotron radiation extend to the Lorentz non-invariant situation. This caused some controversy in the literature [78]. Moreover, an assessment of such assumptions requires the introduction of specific dynamical models. One of them is the Myers-Pospelov (MP) effective theory at the classical level, which parameterizes LIV using dimension five operators Ref. 14. A detailed description of synchrotron radiation in this model has been presented in Refs. [52], which we review in this subsection and that has motivated the general point of view summarized in subsection 4.4 given in more detail in Ref. 53.

The study of radiation in LIV electrodynamics constitutes an interesting problem on its own whose resolution will subsequently allow the use of additional observational information to put bounds upon the correction parameters. For example we have in mind polarization measurements from cosmological sources. The case of gamma ray bursts has recently become increasingly relevant [79, although it is still at a controversial stage 80.

Our calculation of synchrotron radiation in modified electrodynamics rest heavily on previous work reported in Refs. 81, 82, 83, 84, 85].

A partial list of previous studies in electrodynamics incorporating LIV via dimension three and four operators is given in Ref. 86

\section{Synchrotron radiation in the Myers-Pospelov model}

This model parameterizes LIV using dimension five operators both in the matter and electromagnetic sectors. There is also a preferred frame four velocity $V^{\mu}$, which is not a dynamical field. As usual the model exhibits passive (observer) Lorentz covariance, which means that the fields and the four-velocity $V^{\mu}$ transform as tensors when going from one observer frame to another. On the other hand, in each frame we violate active (particle) Lorentz transformations; that is to say we have the non-dynamical physical field $V^{\mu}$ in the action, in analogy to the physics going on in the presence of an external magnetic field which violates active rotational invariance, for example.

\section{The charge sector}

The dynamics of a classical charged particle of mass $\mu$ can be obtained from the action for a scalar charged field. In this case the Myers-Pospelov action is

$$
S_{M P}=\int d^{4} x\left[\partial_{\mu} \varphi^{*} \partial^{\mu} \varphi-\mu^{2} \varphi^{*} \varphi+i \tilde{\eta} \varphi^{*}(V \cdot \partial)^{3} \varphi\right],
$$

with the notation $V \cdot \partial=V^{\mu} \partial_{\mu}$. In momentum space, where we write $\varphi(x)=\varphi_{0} \exp i\left(p^{0} t-\mathbf{p} \cdot \mathbf{x}\right)$ and in the reference frame where $V^{\alpha}=(1, \mathbf{0})$, the modified dispersion relation becomes 


$$
\left(p^{0}\right)^{2}+\tilde{\eta}\left(p^{0}\right)^{3}=\mathbf{p}^{2}+\mu^{2} .
$$

To make contact with the results in Ref. [15] it is necessary to rewrite $\tilde{\eta}=-\eta / M, \eta<0$, where $\eta$ is a dimensionless constant and $M$ is a mass scale characterizing the Lorentz symmetry breaking, which is usually, but not necessarily, identified with the Plank mass. The equation (91) is an exact relation in $\tilde{\eta}$. From here we obtain the Hamiltonian for a massive particle to second order in $\tilde{\eta}$

$$
p^{0}=H=\left(\mathbf{p}^{2}+\mu^{2}\right)^{1 / 2}-\frac{1}{2} \tilde{\eta}\left(\mathbf{p}^{2}+\mu^{2}\right)+\frac{5}{8} \tilde{\eta}^{2}\left(\mathbf{p}^{2}+\mu^{2}\right)^{3 / 2}+O\left(\tilde{\eta}^{3}\right) .
$$

In the following we consider the interaction of a particle having charge $q$ with a static constant magnetic field $\mathbf{B}=\boldsymbol{\nabla} \times \boldsymbol{A}$. The standard minimal coupling produces the Hamiltonian

$$
\begin{aligned}
H= & {\left[\left(\mathbf{p}-\frac{q}{c} \mathbf{A}\right)^{2}+\mu^{2}\right]^{1 / 2}-\frac{1}{2} \tilde{\eta}\left[\left(\mathbf{p}-\frac{q}{c} \mathbf{A}\right)^{2}+\mu^{2}\right] } \\
& +\frac{5}{8} \tilde{\eta}^{2}\left[\left(\mathbf{p}-\frac{q}{c} \mathbf{A}\right)^{2}+\mu^{2}\right]^{3 / 2},
\end{aligned}
$$

to order $O\left(\tilde{\eta}^{3}\right)$. Here $c=3 \times 10^{10} \mathrm{~cm} \mathrm{~s}^{-1}$ denotes the uncorrected velocity of light in vacuum. In the following we set $c=1$. Observe that the dispersion relation (91) provides the exact inversion

$$
(\mathbf{p}-q \mathbf{A})^{2}=(1+\tilde{\eta} E) E^{2}-\mu^{2},
$$

with $E$ being the energy of the particle. The Hamilton equations arising from (93) yield the acceleration

$$
\ddot{\mathbf{r}}=\frac{q}{E}\left(1-\frac{3}{2} \tilde{\eta} E+\frac{9}{4} \tilde{\eta}^{2} E^{2}\right)(\mathbf{v} \times \mathbf{B}) .
$$

As in the usual case, this means that the magnitude $|\mathbf{v}|$ of the particle velocity is constant and that the projection of the trajectory in a plane perpendicular to $\mathbf{B}$ is a circular orbit with a Larmor frequency

$$
\omega_{0}=\frac{|q| B}{E}\left(1-\frac{3}{2} \tilde{\eta} E+\frac{9}{4} \tilde{\eta}^{2} E^{2}\right) .
$$

In general the motion is an helix with pitch angle (the angle between the velocity and the magnetic field) $\alpha$. We restrict ourselves to the motion in the plane perpendicular to the magnetic field, i.e. $\alpha=\pi / 2$. The solution to the equations of motion can be written as

$$
\mathbf{r}(t)=\left(\frac{\beta}{\omega_{0}} \cos \omega_{0} t, \frac{\beta}{\omega_{0}} \sin \omega_{0} t, 0\right),
$$


where we emphasize that we are using the standard definition $\beta=|\mathbf{v}| / c$. The velocity is

$$
\mathbf{v}(t)=\left(-\beta \sin \omega_{0} t, \beta \cos \omega_{0} t, 0\right) .
$$

The equation (97) identifies $R=\beta / \omega_{0}$ as the Larmor radius of the orbit. The Lorentz factor $\gamma$ is given by

$$
\frac{1}{\gamma^{2}}=1-\beta^{2}=\frac{\mu^{2}}{E^{2}}\left[1+2 \frac{\tilde{\eta} E^{3}}{\mu^{2}}-\frac{15}{4} \frac{\tilde{\eta}^{2} E^{4}}{\mu^{2}}+O\left(\tilde{\eta}^{3}\right)\right],
$$

where the range of energies to be considered is such that $\frac{\mu}{E}<<1, \tilde{\eta} E<<1$.

According to the preceding analysis, the current for a charged particle moving in the circular motion is

$$
\mathbf{j}(t, \mathbf{r})=q \delta^{3}(\mathbf{r}-\mathbf{r}(t)) \mathbf{v}(t),
$$

where $\mathbf{r}(t)$ and $\mathbf{v}(t)$ are given in Eqs. (97) and (98) respectively.

The electromagnetic sector

The corresponding action is 14.

$$
S_{\text {photon }}=\int d^{4} x\left[-\frac{1}{4} F_{\mu \nu} F^{\mu \nu}-4 \pi J^{\mu} A_{\mu}+\tilde{\xi}\left(V^{\alpha} F_{\alpha \delta}\right)\left(V^{\nu} \partial_{\nu}\right)\left(V_{\beta} \tilde{F}^{\beta \delta}\right)\right] .
$$

We choose to work in the rest frame $V^{\mu}=(1,0)$ where the modified Maxwell equations are $(c=1)$

$$
\begin{array}{ll}
\nabla \cdot \mathbf{B}=0, & \nabla \times \mathbf{E}+\frac{\partial \mathbf{B}}{\partial t}=0, \\
\nabla \cdot \mathbf{E}=4 \pi \rho, & \left.-\frac{\partial \mathbf{E}}{\partial t}+\nabla \times \mathbf{B}+\tilde{\xi} \partial_{0}\left(-\nabla \times \mathbf{E}+\partial_{0} \mathbf{B}\right)\right)=4 \pi \mathbf{j} .
\end{array}
$$

For future purposes it is convenient to define $\tilde{\xi}=\xi / M$, where $\xi$ is a dimensionless parameter. Introducing the standard potential fields $A^{\mu}=(\phi, \mathbf{A})$ in the Coulomb gauge we have

$$
\begin{aligned}
\phi & =-4 \pi \frac{1}{\nabla^{2}} \rho \\
\frac{\partial^{2} \mathbf{A}}{\partial t^{2}}-\nabla^{2} \mathbf{A}+2 \tilde{\xi} \nabla \times \frac{\partial^{2} \mathbf{A}}{\partial t^{2}} & =4 \pi\left(\mathbf{j}-\nabla \frac{1}{\nabla^{2}} \nabla \cdot \mathbf{j}\right) \equiv 4 \pi \mathbf{j}_{T},
\end{aligned}
$$

where the electric and magnetic fields reduce to

$$
\mathbf{E}=-\frac{\partial \mathbf{A}}{\partial t}, \quad \mathbf{B}=\nabla \times \mathbf{A},
$$

in the radiation approximation. 
The energy momentum tensor $T_{\mu \nu}$ for this modified electrodynamics is given by

$$
\begin{gathered}
T_{0}^{0}=\frac{1}{8 \pi}\left(\mathbf{E}^{2}+\mathbf{B}^{2}\right)-\frac{\tilde{\xi}}{4 \pi} \mathbf{E} \cdot \frac{\partial \mathbf{B}}{\partial t}, \\
\mathbf{S}=\frac{1}{4 \pi} \mathbf{E} \times \mathbf{B}-\frac{\tilde{\xi}}{4 \pi} \mathbf{E} \times \frac{\partial \mathbf{E}}{\partial t},
\end{gathered}
$$

which are exact expressions in $\tilde{\xi}$ and satisfy the usual conservation equation outside the sources.

To solve the equation of motion for A, Eq. (104), we can go to the momentum space with the convention

$$
F(t, \mathbf{r})=\int \frac{d^{4} k}{(2 \pi)^{4}} e^{-i \omega t+i \mathbf{k} \cdot \mathbf{r}} F(\omega, \mathbf{k}) .
$$

The different types of Fourier transforms are specified by the corresponding arguments. For example, if $F(t, \mathbf{r})$ denotes the function in space-time, $F(\omega, \mathbf{r})$ denotes the Fourier transformed function to frequency space, while $F(\omega, \mathbf{k})$ denotes the Fourier transformed function to frequency and momentum spaces. In this way Eq. (104) reduces to

$$
\left(-\omega^{2}+k^{2}-2 i \tilde{\xi} \omega^{2} \mathbf{k} \times\right) \mathbf{A}(\omega, \mathbf{k})=4 \pi \mathbf{j}_{T}(\omega, \mathbf{k}) .
$$

This equation can be diagonalized using the circular polarization basis, with $\lambda= \pm 1$, giving

$$
\left(-\omega^{2}+k^{2} \mp 2 \tilde{\xi} \omega^{2} k\right) \mathbf{A}^{ \pm}(\omega, \mathbf{k})=4 \pi \mathbf{j}_{T}^{ \pm}(\omega, \mathbf{k}) .
$$

The components $C_{i}^{\lambda}$ of any vector $\boldsymbol{C}$ in the polarization basis associated to the direction $\boldsymbol{k}$ are

$$
C_{i}^{\lambda}=P_{i k}^{\lambda} C_{k}, \quad P_{i k}^{\lambda}=\frac{1}{2}\left(\delta_{i k}-\hat{k}_{i} \hat{k}_{k}+\lambda i \epsilon_{i j k} \hat{k}_{j}\right) .
$$

The simplest way to proceed is by introducing the total retarded Green function

$$
\left[G_{r e t}(\omega, \mathbf{k})\right]_{i k}=\left.\sum_{\lambda} P_{i k}^{\lambda} \frac{1}{k^{2}-\lambda 2 \tilde{\xi} \omega^{2} k-\omega^{2}}\right|_{\omega \rightarrow \omega+i \epsilon} .
$$

and to calculate

$$
\left[G_{r e t}\left(\omega, \boldsymbol{r}-\boldsymbol{r}^{\prime}\right)\right]_{i k}=\int \frac{d^{3} \mathbf{k}}{(2 \pi)^{3}} e^{i \mathbf{k} \cdot\left(\mathbf{r}-\mathbf{r}^{\prime}\right)}\left[G_{r e t}(\omega, \mathbf{k})\right]_{i k} .
$$

After some rearrangements the final integration over $d k$ can be performed by the method of residues in the complex plane. Finally one can identify back the polarization components of the total Green function as 


$$
G_{r e t}^{\lambda}\left(\omega, \mathbf{r}-\mathbf{r}^{\prime}\right)=\frac{1}{4 \pi R} \frac{n_{\lambda}(z)}{\sqrt{1+z^{2}}} e^{i n_{\lambda}(z) \omega R},
$$

where $R=\left|\mathbf{r}-\mathbf{r}^{\prime}\right|$. Here we have introduced the polarization-dependent refraction index $n_{\lambda}(z)$

$$
n_{\lambda}(z)=\sqrt{1+z^{2}}+\lambda z, \quad z=\tilde{\xi} \omega
$$

In this way, the fields $\mathbf{A}^{\lambda}$ in Eq. (110) have well defined phase velocities $v_{\lambda}=1 / n_{\lambda}(z)$ and this situation can be described as the propagation of photons in a dispersive birefringent medium.

The Green functions (114) determine the corresponding potentials with the standard replacements $1 / R \simeq 1 /|\mathbf{r}| \equiv 1 / r$ in the denominator, together with the following expansion of the phase $n(\lambda z) \omega R$

$$
n_{\lambda}(z) \omega\left|\mathbf{r}-\mathbf{r}^{\prime}\right| \simeq \omega r\left[1-\frac{\hat{\mathbf{n}} \cdot \mathbf{r}^{\prime}}{r}+\lambda \tilde{\xi} \omega-\lambda \tilde{\xi} \omega \frac{\hat{\mathbf{n}} \cdot \mathbf{r}^{\prime}}{r}+\frac{1}{2}\left(\frac{r^{\prime}}{r}\right)^{2}\right]
$$

where $\hat{\mathbf{n}}=\mathbf{r} / r$ is the direction of observation. Notice that we are interested in the radiation approximation of the phase (116), which means that the subdominant terms of order $\left(r^{\prime} / r\right)^{2}$ or higher are neglected. Consistency demands that the terms proportional to the LIV parameter $\tilde{\xi}$ are larger than the neglected one in order to properly include them in this phase. Our general results are presented in this full far-field approximation.

Using (114) we finally get

$$
\mathbf{A}^{\lambda}(\omega, \mathbf{r})=\frac{1}{r} \frac{n_{\lambda}(z)}{\sqrt{1+z^{2}}} e^{i n_{\lambda}(z) \omega r} \mathbf{j}^{\lambda}\left(\omega, \mathbf{k}_{\lambda}\right)
$$

in the radiation approximation. The fields $\mathbf{A}^{+}(\omega, \mathbf{r})$ and $\mathbf{A}^{-}(\omega, \mathbf{r})$ correspond to right and left circular polarization respectively. Let us emphasize that the momenta

$$
\mathbf{k}_{\lambda}=n_{\lambda}(z) \omega \hat{\mathbf{n}}
$$

in Eq. (117) are fixed in terms of the frequency and the direction of observation. The full vector potential is given by the superposition $\boldsymbol{A}(\omega, \mathbf{r})=$ $\boldsymbol{A}^{+}(\omega, \mathbf{r})+\boldsymbol{A}^{-}(\omega, \mathbf{r})$. Hence the electric and magnetic fields are

$$
\begin{aligned}
& \mathbf{B}(\omega, \mathbf{r})=\frac{1}{r} \frac{\omega}{\sqrt{1+z^{2}}} \sum_{\lambda= \pm} \lambda n_{\lambda}^{2}(z) e^{i n_{\lambda}(z) \omega r} \mathbf{j}^{\lambda}\left(\omega, \mathbf{k}_{\lambda}\right), \\
& \mathbf{E}(\omega, \mathbf{r})=\frac{1}{r} \frac{i \omega}{\sqrt{1+z^{2}}} \sum_{\lambda= \pm} n_{\lambda}(z) e^{i n_{\lambda}(z) \omega r} \mathbf{j}^{\lambda}\left(\omega, \mathbf{k}_{\lambda}\right) .
\end{aligned}
$$

Note that, contrary to the standard case where $\hat{\mathbf{n}} \times \mathbf{E} \propto \mathbf{B}$, here we have

$$
\hat{\mathbf{n}} \times \mathbf{E}(\omega, \mathbf{r})=\frac{1}{\sqrt{1+z^{2}}}[\mathbf{B}(\omega, \mathbf{r})+i z \mathbf{E}(\omega, \mathbf{r})] .
$$


The angular distribution of the power spectrum is defined as

$$
\frac{d^{2} P(T)}{d \omega d \Omega},
$$

where $P(T)$ is the radiated power at time $T$ into the solid angle $d \Omega$. We can compute the total energy emitted in terms of the Poynting vector (107)

$$
E=\int_{-\infty}^{\infty} d t \mathbf{n} \cdot \mathbf{S}(t, \mathbf{r}) r^{2} d \Omega .
$$

This last expression can be rewritten introducing the Fourier transform of the Poynting vector,

$$
E=\int_{0}^{\infty} d \omega \int d \Omega \frac{d^{2} E}{d \Omega d \omega}=\int_{0}^{\infty} \frac{d \omega}{2 \pi}[\mathbf{n} \cdot \mathbf{S}(\omega, \mathbf{r})+\mathbf{n} \cdot \mathbf{S}(-\omega, \mathbf{r})] r^{2} d \Omega,
$$

and allows us to obtain the angular distribution of the energy spectrum

$$
\frac{d^{2} E}{d \Omega d \omega}=\frac{r^{2}}{2 \pi}[\mathbf{n} \cdot \mathbf{S}(\omega, \mathbf{r})+\mathbf{n} \cdot \mathbf{S}(-\omega, \mathbf{r})]
$$

from where the angular distribution of the power spectrum can be identified as

$$
\frac{d^{2} E}{d \Omega d \omega}=\int_{-\infty}^{+\infty} d T \frac{d^{2} P(T)}{d w d \Omega}
$$

Writing the Poynting vector (107) in terms of the polarized potentials we obtain the intermediate result

$$
\frac{d^{2} E}{d \Omega d \omega}=\frac{r^{2} \omega^{2}}{4 \pi^{2}} \sqrt{1+z^{2}}\left[\mathbf{A}_{-}(-\omega, \mathbf{r}) \cdot \mathbf{A}_{+}(\omega, \mathbf{r})+\mathbf{A}_{-}(\omega, \mathbf{r}) \cdot \mathbf{A}_{+}(-\omega, \mathbf{r})\right] .
$$

Next we express the products $\mathbf{A}_{\mp}(-\omega, \mathbf{r}) \cdot \mathbf{A}_{ \pm}(\omega, \mathbf{r})$ in terms of the current $\mathbf{j}(\omega, \mathbf{k})$ via the relation (117). Using the properties of the fields in the polarization basis, together with the general relation $j_{k}(-\omega,-\mathbf{k})=j_{k}^{*}(\omega, \mathbf{k})$, we obtain

$$
\begin{aligned}
\frac{d^{2} E}{d \Omega d \omega}= & \frac{1}{4 \pi^{2}} \frac{\omega^{2}}{1+z^{2}}\left[n_{+}^{2}(z) j_{k}^{*}\left(\omega, \mathbf{k}_{+}\right) P_{k r}^{+} j_{r}\left(\omega, \mathbf{k}_{+}\right)\right. \\
& \left.+n_{-}^{2}(z) j_{k}^{*}\left(\omega, \mathbf{k}_{-}\right) P_{k r}^{-} j_{r}\left(\omega, \mathbf{k}_{-}\right)\right] .
\end{aligned}
$$

In order to identify the angular distribution of the power spectrum we need to introduce the time dependence via the corresponding inverse Fourier transform. Each contribution in Eq. (127) is of the type

$$
C(\omega)=j_{k}^{*}(\omega, \mathbf{k}) X_{k r} j_{r}(\omega, \mathbf{k})=\int_{-\infty}^{+\infty} d t d t^{\prime} e^{-i \omega\left(t-t^{\prime}\right)} j_{k}^{*}(t, \mathbf{k}) X_{k r} j_{r}\left(t^{\prime}, \mathbf{k}\right) .
$$


Changing to new time variables $\tau=t-t^{\prime}$ and $T=\left(t+t^{\prime}\right) / 2$ we get

$$
C(\omega)=\int_{-\infty}^{+\infty} d T \int_{-\infty}^{\infty} d \tau e^{-i \omega \tau} j_{k}^{*}(T+\tau / 2, \mathbf{k}) \quad X_{k r} j_{r}(T-\tau / 2, \mathbf{k}) .
$$

Inserting this last relation in Eq. (127) and comparing with Eq. (125) we obtain the final expression for the angular distribution of the radiated power spectrum

$$
\begin{aligned}
\frac{d^{2} P(T)}{d \omega d \Omega}= & \frac{1}{4 \pi^{2}} \frac{\omega^{2}}{\sqrt{1+z^{2}}} \int_{-\infty}^{\infty} d \tau e^{-i \omega \tau} \times \\
& \sum_{\lambda} n_{\lambda}^{2}(z) j_{k}^{*}\left(T+\tau / 2, \mathbf{k}_{\lambda}\right) P_{k r}^{\lambda} j_{r}\left(T-\tau / 2, \mathbf{k}_{\lambda}\right),
\end{aligned}
$$

as the sum of the contributions of both circular polarizations.

\section{Synchrotron radiation}

This corresponds to the choice (98) for the velocity of the current (100) in the general expression (130). Following the method of Ref. 82 the time-averaged angular distribution of the radiated power spectrum is

$$
\begin{aligned}
& \left\langle\frac{d^{2} P(T)}{d \omega d \Omega}\right\rangle=\sum_{\lambda=+,-} \sum_{m=0}^{\infty} \delta\left(\omega-\omega_{m}\right) \frac{d P_{m, \lambda}}{d \Omega}, \quad \omega_{m}=m \omega_{0}, \quad z_{m}=\tilde{\xi} \omega_{m}, \\
& \frac{d P_{m, \lambda}}{d \Omega}=\frac{\omega_{m}^{2} q^{2}}{4 \pi} \frac{1}{\sqrt{1+z_{m}^{2}}}\left[\lambda \beta n_{\lambda}\left(z_{m}\right) J_{m}^{\prime}\left(W_{\lambda m}\right)+\cot \theta J_{m}\left(W_{\lambda m}\right)\right]^{2},
\end{aligned}
$$

written as a sum over the contribution of the harmonics $\omega_{m}$. Here, the average \langle\rangle is taken with respect to the macroscopic time $T$ and $J_{m}, J_{m}^{\prime}$ denote the Bessel functions and their derivatives respectively. The argument of the Bessel functions is $W_{\lambda m}=m n_{\lambda}\left(z_{m}\right) \beta \sin \theta$.

We also have calculated the total averaged and integrated power radiated into the $m$-th harmonic

$$
\begin{aligned}
P_{m}= & \frac{q^{2} \beta^{2} \omega_{m}}{R \sqrt{1+z_{m}^{2}}} \sum_{\lambda= \pm} n_{\lambda}\left(z_{m}\right)\left[J_{2 m}^{\prime}\left(2 m n_{\lambda}\left(z_{m}\right) \beta\right)\right. \\
& \left.-\frac{1-\beta^{2} n_{\lambda}^{2}\left(z_{m}\right)}{2 \beta^{2} n_{\lambda}^{2}\left(z_{m}\right)} \int_{0}^{2 m n_{\lambda}\left(z_{m}\right) \beta} d x J_{2 m}(x)\right]
\end{aligned}
$$

which clearly indicates the contribution of each polarization $P_{\lambda m}$. The above result is exact in $z_{m}$ and the parity-violating contribution has vanished after the angular integration.

In Table 3 we present a rough estimation of the relevant parameters associated with some observed cosmological objects. There $r[l . y$.$] is the distance$ 
Table 3. Data of some relevant astrophysical objects

\begin{tabular}{lllccccc}
\hline Object & $r($ l.y. $)$ & $\gamma$ & $B$ (Gauss) & $\omega_{c}(\mathrm{GeV})$ & $\omega_{0}(\mathrm{GeV})$ & $m$ & $\mathrm{~m} / \gamma$ \\
\hline CRAB & $10^{4}$ & $10^{9}$ & $10^{-3}$ & $10^{-1}$ & $10^{-30}$ & $10^{29}$ & $10^{20}$ \\
$(M k n 501)_{p}$ & $10^{8}$ & $10^{11}$ & $10^{2}$ & $10^{4}$ & $10^{-29}$ & $10^{33}$ & $10^{22}$ \\
$(M k n 501)_{e}$ & $10^{8}$ & $10^{11}$ & $10^{-1}$ & $10^{4}$ & $10^{-29}$ & $10^{33}$ & $10^{22}$ \\
GRB 021006 & $10^{10}$ & $10^{5}$ & $10^{4}$ & $10^{-3}$ & $10^{-18}$ & $10^{15}$ & $10^{10}$ \\
\hline
\end{tabular}

of the object to the earth, $\gamma$ is the Lorentz factor of the charged particles at the cutoff frequency, $B[$ Gauss $]$ is the average magnetic field producing the synchrotron radiation, $\omega_{c}[\mathrm{GeV}]$ is the cut-off frequency and $\omega_{0}[\mathrm{GeV}]$ is the Larmor frequency. In all cases the cut-off frequency $\omega_{c}$ has been estimated from the radiation spectrum fitted by a synchrotron model in the corresponding reference. This, together with the magnetic field $B$ allows us to estimate the Lorentz factor

$$
\gamma=2.36 \times 10^{8} \sqrt{\frac{\omega_{c}[\text { GeV }]}{B[\text { Gauss }]} \frac{M}{m_{e}}},
$$

where $M$ is the mass of the charged particle. From the above we further obtain the Larmor frequency

$$
\omega_{0}[G e V]=0.6 \times 10^{-17}\left(\frac{2 m_{e}}{\gamma M}\right) B[\text { Gauss }] .
$$

In the case of CRAB Nebula we adopt the typical values given in Ref. 87]. For Mkn 501 we consider two possible models for synchrotron radiation where the emitter particles are either protons 88 or electrons 89 . In the latter case we use the radius of the orbit $r^{\prime}=1.5 \times 10^{15} \mathrm{~cm}=1 / \omega_{0}$ and the magnetic field to obtain the Lorentz factor. Finally we consider the GRB021206 $(z \approx 1.25)$. According to Ref. [90 this object has a very compact core with a radius of the order of $1 \mathrm{~km}$ and a magnetic field $\approx 10^{12}$ Gauss. The synchrotron emission region is about $10^{8} \mathrm{~km}$ from the core 91, so that we estimate the magnetic field to be $10^{4}$ Gauss using the transport law $B r=$ const.. From Ref. 92 we take the cut-off frequency to be $\omega_{c}=1 \mathrm{MeV}$.

As indicated in Table 3, the radiation of interest is dominated by very high harmonics $10^{15} \leq m \leq 10^{30}$ exhibiting also large ratios of $m / \gamma$, typically in the range $10^{10} \leq m / \gamma \leq 10^{22}$. The corresponding values for $\gamma$ imply also $\beta \approx 1$. In this way, the high harmonics present in the synchrotron radiation of these astrophysical sources together with the values of the $\gamma$ factors of the radiating charges highlight the relevance of the large $m$ and large $\gamma$ limit, with the constraint $(m / \gamma)^{2} \gg 1$, to study the induced Lorentz violating effects.

In a similar way to the standard case 83 , we obtain

$$
P_{\lambda m}=\frac{q^{2} m \omega_{0}}{\sqrt{3} \pi R} \frac{1}{1+n^{2}\left(\lambda z_{m}\right)}\left\{\int_{m / \tilde{m}_{\lambda c}}^{\infty} d x\left(\frac{3}{2 \tilde{m}_{\lambda c}}\right)^{2 / 3} K_{5 / 3}(x)\right.
$$




$$
\left.-2\left(\frac{3}{2 \tilde{m}_{\lambda c}}\right)^{4 / 3} K_{2 / 3}\left(\frac{m}{\tilde{m}_{c}}\right)\right\},
$$

for the integrated power in the $m^{\text {th }}$ harmonic. Here $K_{p / q}$ denote the Macdonald functions (Bessel functions of fractional order). A first consequence of this approximation is the appearance of the cutoff frequency $\omega_{\lambda c}=\tilde{m}_{\lambda_{c}} \omega_{0}$ with

$$
\tilde{m}_{\lambda c}=\frac{3}{2}\left[1-\beta^{2}(E) n_{\lambda}^{2}\left(z_{m}\right)\right]^{-3 / 2} .
$$

This name arises because for $m>\tilde{m}_{\lambda c}$ the total power decreases as

$$
P_{\lambda m} \approx \exp \left(-m / \tilde{m}_{\lambda c}\right) .
$$

Within the same large- $m$ approximation, the integrated power in the $m$-th harmonic can be expanded to second order in $\tilde{\xi}$ yielding

$$
\begin{aligned}
P_{m}= & \frac{q^{2} \omega}{\sqrt{3} \pi R \gamma^{2}}\left[\frac{m_{c}}{m} \kappa\left(\frac{m}{m_{c}}\right)-\frac{2}{\gamma^{2}} K_{2 / 3}\left(\frac{m}{m_{c}}\right)\right. \\
& \left.+2\left(\frac{\tilde{\xi} m \omega \beta}{\gamma}\right)^{2} K_{2 / 3}\left(\frac{m}{m_{c}}\right)\right],
\end{aligned}
$$

where $m_{c}=3 \gamma^{3} / 2$ and $\kappa(x)=x \int_{x}^{\infty} d y K_{5 / 3}(y)$ is the so called bremsstrahlung function.

Let us notice the appearance of the combination $\tilde{\xi} \omega m / \gamma=\xi\left(\omega / M_{P}\right)(m / \gamma)$ as the expansion parameter in (137). Here we take $M=M_{P}$. As can be seen from Table 3 this is not necessarily a small number, which signals the possibility that such corrections might be relevant in setting bounds upon $\tilde{\xi}$. This rather unexpected effect is due to the amplifying factor $m / \gamma$. Similar results have been obtained in calculations of the synchrotron radiation spectra in the context of non-commutative electrodynamics 93 .

Another possibility for observable effects due to $\tilde{\xi}$ is to look at the averaged degree of circular polarization

$$
\Pi_{\odot}=\frac{\left\langle P_{+}(\omega)-P_{-}(\omega)\right\rangle}{\left\langle P_{+}(\omega)+P_{-}(\omega)\right\rangle}
$$

where $P_{\lambda}(\omega)$ is the total power distribution per unit frequency and polarization $\lambda$, so that $P_{\lambda}(\omega)=P_{m \lambda} / \omega_{0}$. The average here is calculated with respect to an energy distribution of the relativistic electrons, which we take to be $N(E) d E=C E^{-p} d E$, in some energy range $E_{1}<E<E_{2}$, chosen as $E_{1}=0$ and $E_{2} \rightarrow \infty$ for simplicity. The result is

$$
\Pi_{\odot}=\tilde{\xi} \omega\left(\frac{\mu \omega}{q B}\right) \Pi(p),
$$




$$
\Pi(p)=\frac{(p-3)(3 p-1)}{3(3 p-7)} \frac{(p+1)}{(p-1)} \frac{\Gamma\left(\frac{p}{4}+\frac{13}{12}\right) \Gamma\left(\frac{p}{4}+\frac{5}{12}\right)}{\Gamma\left(\frac{p}{4}+\frac{19}{12}\right) \Gamma\left(\frac{p}{4}+\frac{11}{12}\right)}, \quad p>7 / 3 .
$$

Again, we have the presence of an amplifying factor in Eq. (139), given by $(\mu \omega / q B)$, which is independent of the form of $\Pi(p)$ and not necessarily a small number. An estimation of this factor in the zeroth-order approximation $(\tilde{\xi}=0=\tilde{\eta})$, which is appropriate in (139), yields $(\mu \omega / q B)=\omega /\left(\omega_{0} \gamma\right)=m / \gamma$. The expression (139) is analogous to the well-known average of the degree of linear polarization $\Pi_{L I N}=(p+1) /(p+7 / 3)$, under the same energy distribution for the electrons 94 .

Finally in Table 4 we have estimated the contributions to the different pieces in the phase (116) of the Green function, for each astrophysical object. Here we have set $\xi=1$ and $M=M_{P}$. The extreme case is

Table 4. The far field approximation

\begin{tabular}{llccc}
\hline Object & $r^{\prime} / r$ & $\omega_{c} / M_{P}$ & $\left(r^{\prime} / r\right)\left(\omega_{c} / M_{P}\right)$ & $\left(r^{\prime} / r\right)^{2}$ \\
\hline CRAB & $10^{-6}$ & $10^{-20}$ & $10^{-26}$ & $10^{-12}$ \\
Mkn 501 & $10^{-11}$ & $10^{-15}$ & $10^{-26}$ & $10^{-22}$ \\
GRB 021006 & $10^{-24}$ & $10^{-22}$ & $10^{-46}$ & $10^{-48}$ \\
\hline
\end{tabular}

$$
|\tilde{\xi} \omega| \frac{r^{\prime}}{r}<|\tilde{\xi} \omega|<\left(\frac{r^{\prime}}{r}\right)^{2}
$$

where all the dependence on $\tilde{\xi}$ is negligible in the phase, which reduces to

$$
(\lambda z) \omega\left|\boldsymbol{r}-\boldsymbol{r}^{\prime}\right| \simeq \omega\left(r-\hat{n} \cdot \boldsymbol{r}^{\prime}\right) .
$$

This corresponds to the CRAB nebulae case, where the assumptions made in Ref. 15] are readily verified. A detailed discussion of synchrotron radiation in the Myers-Pospelov model can be found in the third reference [52].

\subsection{General point of view of LIV radiation}

Three paradigmatic examples of Lorentz violating electrodynamics are given by the effective theories proposed by Gambini and Pullin (GP) [30, Ellis et al. (EMN) 28, and Myers and Pospelov (MP) [14. They can be written in the general form of Maxwell equations

$$
\begin{array}{ll}
\nabla \cdot \mathbf{D}=4 \pi \rho, & \nabla \cdot \mathbf{B}=0, \\
\nabla \times \mathbf{E}=-\frac{\partial \mathbf{B}}{\partial t}, & \nabla \times \mathbf{H}=\frac{\partial \mathbf{D}}{\partial t}+4 \pi \mathbf{j},
\end{array}
$$

with corresponding constitutive relations 


$$
\mathbf{D}=\mathbf{D}(\mathbf{E}, \mathbf{B}), \quad \mathbf{H}=\mathbf{H}(\mathbf{E}, \mathbf{B}),
$$

which we next present in detail for each case, after reviewing the corresponding equations. Let us recall that the above equations (143) and (144) imply charge conservation $\partial \rho / \partial t+\nabla \cdot \mathbf{j}=0$, independently of the constitutive equations (145). In an abuse of notation we have denoted by $\tilde{\xi}$ the electromagnetic LIV parameter for all models in the sequel.

\section{Gambini-Pullin Electrodynamics}

The Maxwell equations for this case are

$$
\begin{array}{ll}
\nabla \cdot \mathcal{B}=0, & \nabla \times(\mathcal{E}+2 \tilde{\xi} \nabla \times \mathcal{E})+\frac{\partial \mathcal{B}}{\partial t}=0, \\
\nabla \cdot \mathcal{E}=4 \pi \rho, & \nabla \times(\mathcal{B}+2 \tilde{\xi} \nabla \times \mathcal{B})-\frac{\partial \mathcal{E}}{\partial t}=4 \pi \mathbf{j},
\end{array}
$$

where the electric and magnetic fields are identified from the homogeneous equation as

$$
\mathbf{E}=\mathcal{E}+2 \tilde{\xi} \nabla \times \mathcal{E}, \quad \mathbf{B}=\mathcal{B} .
$$

From the inhomogeneous equations we obtain

$$
\mathbf{D}=\mathcal{E}, \quad \mathbf{H}=\mathcal{B}+2 \tilde{\xi} \nabla \times \mathcal{B},
$$

which together with the constitutive relations

$$
\mathbf{D}+2 \tilde{\xi} \nabla \times \mathbf{D}=\mathbf{E}, \quad \mathbf{H}=\mathbf{B}+2 \tilde{\xi} \nabla \times \mathbf{B},
$$

leave the equations in the required form. These equations define the corresponding functions stated in (145). In momentum space we have

$$
\mathbf{D}=\frac{1}{1+4 \tilde{\xi}^{2} k^{2}}\left(\mathbf{E}-2 i \tilde{\xi} \mathbf{k} \times \mathbf{E}+4 \tilde{\xi}^{2}(\mathbf{k} \cdot \mathbf{E}) \mathbf{k}\right), \quad \mathbf{H}=\mathbf{B}+2 i \tilde{\xi} \mathbf{k} \times \mathbf{B} .
$$

The admixture of vectors and axial vectors in the constitutive relations precludes the parity violation exhibited by the model, together with the presence of birefringence.

Ellis et al. Electrodynamics

In this case the modified Maxwell equations are

$$
\begin{aligned}
& \nabla \cdot \mathbf{B}=0, \quad \nabla \times \mathbf{E}+\frac{\partial \mathbf{B}}{\partial t}=0, \\
& \nabla \cdot \mathbf{E}+\mathbf{u} \cdot \frac{\partial \mathbf{E}}{\partial t}=4 \pi \rho_{e f f}=4 \pi(\rho-\mathbf{u} \cdot \mathbf{j}), \\
& \nabla \times \mathbf{B}-\left(1-\mathbf{u}^{2}\right) \frac{\partial \mathbf{E}}{\partial t}+\mathbf{u} \times \frac{\partial \mathbf{B}}{\partial t}+(\mathbf{u} \cdot \nabla) \mathbf{E}=4 \pi \mathbf{j}_{e f f} \\
& =4 \pi(\mathbf{j}+\mathbf{u}(\rho-\mathbf{u} \cdot \mathbf{j})),
\end{aligned}
$$


which can be written in the form (143 144) via the constitutive relations [28] $\mathbf{H}=\mathbf{B}-f(\omega) \mathbf{k} \times \mathbf{E}, \quad \mathbf{D}=\left(1-f^{2}(\omega) k^{2}\right) \mathbf{E}+f^{2}(\omega) \mathbf{k}(\mathbf{k} \cdot \mathbf{E})-f(\omega) \mathbf{k} \times \mathbf{B}$,

where we have assumed that $\mathbf{u}=f(\omega) \mathbf{k}$ in momentum space. Taking $\mathbf{u}$ as a vector, this model conserves parity and shows no birefringence.

\section{Myers-Pospelov Electrodynamics}

This case corresponds to the equations (102). From the last one we can infer the constitutive relations

$$
\mathbf{H}=\mathbf{B}-\tilde{\xi} \partial_{0} \mathbf{E}, \quad \mathbf{D}=\mathbf{E}-\tilde{\xi} \partial_{0} \mathbf{B},
$$

which produce

$$
\nabla \cdot \mathbf{E}=\nabla \cdot \mathbf{D},
$$

leaving the third Eq. (102) in desired form. Similarly to the GP case, this model violates parity. In momentum space Eqs. (156) become

$$
\mathbf{H}=\mathbf{B}+i \tilde{\xi} \omega \mathbf{E}, \quad \mathbf{D}=\mathbf{E}+i \tilde{\xi} \omega \mathbf{B}
$$

The above constitutive relations in the three representative models involve linear relations among the fields and can be summarized, in momentum space, as the local relations

$$
\begin{aligned}
& D_{i}(\omega, \mathbf{k})=\alpha_{i j}(\omega, \mathbf{k}) E_{i}(\omega, \mathbf{k})+\rho_{i j}(\omega, \mathbf{k}) B_{j}(\omega, \mathbf{k}), \\
& H_{i}(\omega, \mathbf{k})=\beta_{i j}(\omega, \mathbf{k}) B_{i}(\omega, \mathbf{k})+\sigma_{i j}(\omega, \mathbf{k}) E_{j}(\omega, \mathbf{k}),
\end{aligned}
$$

where the corresponding momentum dependent coefficients can be read from the equations (151), (155), and (158). Equations (159) are the most general expressions in which any pair of linear constitutive relations can be ultimately written, which allow to express the fields $\mathbf{D}, \mathbf{H}$ in terms of $\mathbf{E}, \mathbf{B}$.

\section{Parameterization of the constitutive relations}

Let us consider Maxwell equations in momentum space

$$
\begin{array}{ll}
\mathbf{k} \cdot \mathbf{B}(\omega, \mathbf{k})=0, & \mathbf{k} \times \mathbf{E}(\omega, \mathbf{k})=\omega \mathbf{B}(\omega, \mathbf{k}), \\
i \mathbf{k} \cdot \mathbf{D}(\omega, \mathbf{k})=4 \pi \rho(\omega, \mathbf{k}), & i \mathbf{k} \times \mathbf{H}(\omega, \mathbf{k})=-i \omega \mathbf{D}(\omega, \mathbf{k})+4 \pi \mathbf{j}(\omega, \mathbf{k}) .
\end{array}
$$

Here we discuss the vacuum situation where the non trivial constitutive relations arise because of LIV effects. Let us take into account corrections up to second order in the LIV parameter $\tilde{\xi}$ and let us assume that we are in a Lorentz frame where we demand invariance under rotations. This would 
correspond to the rest frame $V^{\mu}=(1,0)$ in the MP model, for example. We can always go to an arbitrary frame by means of a passive (observer) Lorentz transformation. In this way we have the general expressions

$$
\begin{array}{ll}
\alpha_{i j}=\alpha_{0} \delta_{i j}+i \alpha_{1} \tilde{\xi} \epsilon_{i r j} k_{r}+\alpha_{2} \tilde{\xi}^{2} k_{i} k_{j}, & \rho_{i j}=\rho_{0} \delta_{i j}+i \rho_{1} \tilde{\xi} \epsilon_{i r j} k_{r}+\rho_{2} \tilde{\xi}^{2} k_{i} k_{j}, \\
\beta_{i j}=\beta_{0} \delta_{i j}+i \beta_{1} \tilde{\xi} \epsilon_{i r j} k_{r}+\beta_{2} \tilde{\xi}^{2} k_{i} k_{j}, & \sigma_{i j}=\sigma_{0} \delta_{i j}+i \sigma_{1} \tilde{\xi} \epsilon_{i r j} k_{r}+\sigma_{2} \tilde{\xi}^{2} k_{i} k_{j},
\end{array}
$$

where $\alpha_{A}, \beta_{A}, \rho_{A}, \sigma_{A}, A=0,1,2$, are scalar functions depending only upon $\omega, k=|\mathbf{k}|$, and $\tilde{\xi}$. The property $\mathbf{k} \cdot \mathbf{B}=0$ sets $\beta_{2}=\rho_{2}=0$ effectively. In vector notation we then have

$$
\begin{aligned}
& \mathbf{D}=\left(\alpha_{0}+\alpha_{2} k^{2} \tilde{\xi}^{2}\right) \mathbf{E}+\left(\rho_{0}+i \alpha_{1} \omega \tilde{\xi}\right) \mathbf{B}+i\left(\rho_{1}-i \alpha_{2} \omega \tilde{\xi}\right) \tilde{\xi} \mathbf{k} \times \mathbf{B}, \\
& \mathbf{H}=\left(\sigma_{0}+\sigma_{2} k^{2} \tilde{\xi}^{2}\right) \mathbf{E}+\left(\beta_{0}+i \sigma_{1} \omega \tilde{\xi}\right) \mathbf{B}+i\left(\beta_{1}-i \sigma_{2} \omega \tilde{\xi}\right) \tilde{\xi} \mathbf{k} \times \mathbf{B},
\end{aligned}
$$

where we have used the second Eq. (160) together with $(\mathbf{k} \cdot \mathbf{E}) \mathbf{k}=\omega(\mathbf{k} \times \mathbf{B})+$ $k^{2} \mathbf{E}$.

The generalized Maxwell equations [53]

Next we substitute (163) in Eqs. (161) to obtain the corresponding equations for $\mathbf{E}$ and $\mathbf{B}$. The result is

$$
\begin{aligned}
& i\left(\alpha_{0}+\alpha_{2} k^{2} \tilde{\xi}^{2}\right)(\mathbf{k} \cdot \mathbf{E})=4 \pi \rho \\
& \begin{aligned}
i\left(\alpha_{0}+\alpha_{2} k^{2} \tilde{\xi}^{2}\right) \omega \mathbf{E}+i\left[\beta_{0}+i\left(\sigma_{1}+\rho_{1}\right) \omega \tilde{\xi}+\alpha_{2} \tilde{\xi}^{2} \omega^{2}\right] \mathbf{k} \times \mathbf{B} \\
+i\left[\left(\sigma_{0}+\rho_{0}\right) \omega+i\left(\alpha_{1} \omega^{2}-\beta_{1} k^{2}\right) \tilde{\xi}\right] \mathbf{B}=4 \pi \mathbf{j}(\omega, \mathbf{k})
\end{aligned}
\end{aligned}
$$

It is convenient to rewrite the inhomogeneous equations in the compact form

$$
\begin{aligned}
i P(\mathbf{k} \cdot \mathbf{E}) & =4 \pi \rho, \\
i \omega P \mathbf{E}+i Q \mathbf{k} \times \mathbf{B}+R \mathbf{B} & =4 \pi \mathbf{j}(\omega, \mathbf{k}),
\end{aligned}
$$

by defining

$$
\begin{aligned}
& P=\alpha_{0}+\alpha_{2} \tilde{\xi}^{2} k^{2}, \quad Q=\beta_{0}+i\left(\sigma_{1}+\rho_{1}\right) \omega \tilde{\xi}+\alpha_{2} \tilde{\xi}^{2} \omega^{2}, \\
& R=\left(\beta_{1} k^{2}-\alpha_{1} \omega^{2}\right) \tilde{\xi}+i\left(\sigma_{0}+\rho_{0}\right) \omega .
\end{aligned}
$$

Now we have only three independent functions which depend on $\omega$ and $k$. The inhomogeneous equations (166) and (167) can be solved by introducing the standard electromagnetic potentials in the Coulomb gauge. The radiation approximation is described in terms of the vector potential $\boldsymbol{A}^{\lambda}$ only, which 
will exhibit polarizations $\lambda= \pm 1$ in the general case and that satisfies the equation

$$
\left[Q k^{2}-P \omega^{2}+\lambda k R\right] \mathbf{A}^{\lambda}=4 \pi \mathbf{j}_{T}^{\lambda}
$$

Here $\mathbf{j}_{T}^{\lambda}$ is the transverse part of the polarized current. The Green function $G^{\lambda}(\omega, k)$ corresponding to the operator in the LHS of (169) satisfies in general a cubic equation in $k$, which determines the dispersion relations for the propagating photon. This means that we have three poles $k_{\lambda}(\omega)$. In fact one of the poles is due to the $\beta_{1}$ factor in the expression for $R$ in Eq. (168) and it is located at $k \sim \xi^{-1}$. Therefore its contribution can be neglected in our effective theory valid for $k \ll \xi^{-1}$. The remaining two poles will deviate little from their position when $\tilde{\xi}=0$ and will allow us to characterize the propagation mode corresponding to each polarization $\lambda$ by a refraction index $n_{\lambda}(\omega)$ to be read from the appropriate dispersion relation in such a way that $n_{\lambda}(\omega)=k_{\lambda}(\omega) / \omega$. The general form of the polarized Green function will be

$$
G^{\lambda}(\omega, \mathbf{r})=\frac{1}{4 \pi r} F^{\lambda}(\omega) e^{i \omega n_{\lambda}(\omega) r} .
$$

Notice that from the birefringent case we can go to the non-birefringent one by taking $n_{+}(\omega)=n_{-}(\omega)=n(\omega)$, in which case $F^{+}(\omega)=F^{-}(\omega)=F(\omega)$.

In the following we make explicit some general properties that must be satisfied by any electrodynamics characterized by a Green function of the type (170). The reality of the electric and magnetic fields leads to the condition

$$
\left[G^{+}(\omega, \mathbf{r})\right]^{*}=G^{-}(-\omega, \mathbf{r}) .
$$

This implies the relations

$$
n_{+}^{*}(\omega)=n_{-}(-\omega), \quad\left[F^{+}(\omega)\right]^{*}=F^{-}(-\omega) .
$$

For a birefringent medium the real and imaginary parts of the refraction index for circular polarization components can contain both $\omega$-even and $\omega$ odd terms, provided that they satisfy the first Eq. (172). In the case of a non-birefringent medium the real part of the refraction index must be even in $\omega$, while the imaginary part must be odd. We can see that the refraction indices for the Myers-Pospelov theory, Eq. (115), satisfy these requirements. A detailed discussion of this generalized point of view is under preparation 95.

\section{Acknowledgements}

The author acknowledges C. Lämmerzhal for his invitation to participate in the $339^{\text {th }}$ WE-Heraeus-Seminar: Special Relativity, will it survive the next 100 years?, as well as for his wonderful hospitality at Potsdam. Partial support from the projects CONACYT-México-40745-F and DGAPA-UNAMIN104503-3; as well as the hospitality of the George P. and Cynthia W. Mitchell Institute for Fundamental Physics, Texas A\&M University are also gratefully acknowledged. 


\section{References}

1. For reviews see for example: C. Rovelli: Loop quantum gravity, Livings Reviews, 1, 1 (1998), URL http://www.livingreviews.org/Articles C. Rovelli: Quantum Gravity, (Cambridge University Press, Cambridge 2004); R. Gambini and J. Pullin: Loops, Knots, Gauge Theories and Quantum Gravity, (Cambridge University Press, Cambridge 1996); C. Beetle and A. Corichi: Bibliography of publications related to classical and quantum gravity in terms of connections and loop variables, gr-qc/9703044

2. For reviews see for example: L. Dolan: TASI Lectures on perturbative string theory and Ramond-Ramond flux. In: String, branes and extra dimensions: TASI 2001, ed by S.S. Gubser and J.D. Lykken (World Scientific, River Edge, N. J. 2004) pp 161-193; R.J. Szabo: BUSSTEPP Lectures on string theory: an introduction to string theory and D-brane dynamics, hep-th/0207142 J. Polchinski: String Theory, Vols I and II, (Cambridge University Press, Cambridge 2000).

3. G. Amelino-Camelia: Nature (London) 398, 216 (1999); D.V. Ahluwalia: Nature (London) 398, 199 (1999); G. Amelino-Camelia: Lect. Notes Phys. 541, 1 (2000); G. Amelino-Camelia, Int. Jour. Mod. Phys. D10, 1 (2001); N.E. Mavromatos: The quest for quantum gravity: testing times for theories?. In: From Particles to the Universe, ed by A. Astbury et al. (World Scientific, Singapore 2001) pp 335-341; J. Ellis: Perspectives in High-Energy Physics. In: Proceedings of the 3rd Latin American Symposium on High-Energy Physics (SILAFAE III), ed by E. Nardi (Institute of Physics, Bristol 2000); G.Z. Adunas, E. Rodriguez-Milla and D.V. Ahluwalia: Phys. Letts. B485, 215 (2000); G.Z. Adunas, E. Rodriguez-Milla and D.V. Ahluwalia: Gen. Rel. Grav. 33, 183 (2001); J. Ellis: Nuovo Cim. 24C, 483 (2001); G. AmelinoCamelia: Mod. Phys. Lett. A17, 899 (2002); S. Sarkar: Mod. Phys. Lett. A17, 1025 (2002); D.V. Ahluwalia: Mod. Phys. Lett. A17, 1135 (2002); G. Amelino-Camelia: contribution to these Proceedings.

4. S.D. Biller et. al.: Phys. Rev. Lett. 83, 2108 (1999).

5. O. Betolami and C.S. Carvalho, Phys. Rev. D61, 103002 (2000); J.M. Carmona and J.L. Cortés: Phys. Lett. B 494, 75 (2000); J.M. Carmona and J.L. Cortés: Phys. Rev. D65, 025006 (2002); C. Lämmerzhal and C. Bordé: Testing the Dirac equation. In: Lecture Notes in Physics 562, ed by C. Lämmerzhal, C.W.F. Everitt and F.W. Hehl (Springer, Heidelberg 2001) pp 463-478; M.P. Haugan and C. Läemmerzhal: Principles of equivalence: their role in gravitation physics and experiments that test them. In: Lecture Notes in Physics 562, ed by C. Lämmerzhal, C.W.F. Everitt and F.W. Hehl (Springer, Heidelberg 2001) pp 195-212; G. Lambiase: Gen. Rel. Grav. 33, 2151 (2001); G. Lambiase: Eur. Phys. J. C19, 553 (2001); G. Amelino-Camelia: Phys. Letts. B528, 181 (2002); G. Lambiase: Class. Quant. Grav. 20, 4213 (2003); G. Lambiase and P. Singh: Phys. Letts. B565, 27 (2003); G. Amelino-Camelia and C. Lämmerzhal: Class. Quant. Grav. 21, 899 (2004); F.W. Stecker: Astropart. Phys. 20, 85 (2003); F.W. Stecker: J. Phys. G29, R47 (2003).

6. C. Rovelli and S. Speziale: Phys. Rev. D67, 064019 (2003).

7. F. Dowker, J. Henson, and R.D. Sorkin: Mod. Phys. Lett. A19, 1829 (2004).

8. V.A. Kostelecký and S. Samuel: Phys. Rev. D39, 683 (1989), V.A. Kostelecký and S. Samuel: Phys. Rev. D40, 1886 (1989); V.A. Kostelecký and R. Potting: 
Nucl. Phys. B359, 545 (1991); V.A. Kostelecký and R. Potting: Phys. Lett. B381, 89 (1996).

9. V.A. Kostelecký and R. Lehnert: Phys. Rev. D63, 065008 (2001).

10. V.W. Hughes, H.G. Robinson and V. Beltrán-López: Phys. Rev. Lett. 4, 342 (1960); R.W.P. Drever: Phil. Mag. 6, 683 (1961).

11. D. Colladay and V.A. Kostelecký: Phys. Rev. D55, 6760 (1997); D. Colladay and V.A. Kostelecký: Phys. Rev. D58, 116002 (1998). For reviews see for example Proceedings of the Meeting on CPT and Lorentz Symmetry, ed by V.A. Kostelecký (World Scientific, Singapore 1999); C.M. Will, The Confrontation between General Relativity and Experiment (Living Reviews in Relativity 4, 2001); Proceedings of the Second Meeting on CPT and Lorentz Symmetry, ed by V. A. Kostelecký (World Scientific, Singapore 2002).

12. J.R. Ellis, J.L. Lopez, N.E. Mavromatos and D.V. Nanopoulos: Phys. Rev. D53, 3846 (1996).

13. V. A. Kostelecký: Phys. Rev. D69, 105009 (2004).

14. R.C. Myers and M. Pospelov: Phys. Rev. Letts. 90, 211601 (2003); R.C. Myers and M. Pospelov: Experimental Challenges of Quantum Gravity. In: Quantum Theory and Symmetries, ed by P.C. Argyres, T.J. Hodges, F. Mansouri, J.J. Scanio, P. Suranyi and L.C.R. Wijewardhana (World Scientific, New Jersey 2004) pp 732-744; A. Pérez and D. Sudarsky: Phys. Rev. Letts. 91, 179101 (2003).

15. T. Jacobson, S. Liberati and D. Mattingly: Nature 424, 1019 (2003).

16. T. Jacobson, S. Liberati and D. Mattingly: Phys. Rev. D67, 124011 (2003); T. Jacobson, S. Liberati and D. Mattingly: Phys. Rev. D66, 081302 (2002); T. Jacobson, S. Liberati, D. Mattingly and F. Stecker: Phys. Rev. Lett. 93, 021101 (2004).

17. C. Lämmerzahl, A. Macías and H. Mueller: Phys. Rev. D71, 025007 (2005) and references therein.

18. A.A. Andrianov and R. Soldati: Phys. Rev. D51, 5961 (1995); A.A. Andrianov and R. Soldati: Phys. Letts. B435, 449 (1998); A.A. Andrianov, P. Giacconi and R. Soldati: JHEP 0202, 030 (2002).

19. G. Amelino-Camelia: Int. J. Mod. Phys. D11, 35 (2002); S. Judes and M. Visser: Phys. Rev. D68, 045001 (2003); A. Agostini, G. Amelino-Camelia and M. Arzano: Class. Quant. Grav. 21, 2179 (2004); D.V. Ahluwalia-Khalilova: Fermions, bosons and locality in special relativity with two invariant scales. In: Proceedings of the 4 th International Conference on Physics Beyond the Standard Model: Beyond the Desert (BEYOND 03), ed by H.V. KlapdorKleingrothaus (Springer, Berlin 2004) pp 503-511; G. Amelino-Camelia, L. Smolin and A. Starodubtsev: Class. Quant. Grav. 21, 3095 (2004); G. Amelino-Camelia: Three perspectives on the quantum gravity problem and their implications for the fate of Lorentz symmetry, gr-qc/0309054

20. J. Magueijo and L. Smolin: Phys. Rev. Lett 88, 190403 (2002); J. Magueijo and L. Smolin: Phys. Rev. D67, 044017 (2003).

21. S. Liberati, S. Sonego and M. Visser: Phys. Rev. D71, 045001 (2005).

22. F. Girelli and E. Livine: Physics of deformed special relativity: relativity principle revisited, gr-qc/0412004

23. L. Smolin: Falsifiable predictions from semiclassical quantum gravity, hep-th/0501091 
24. K. Imilkowska and J. Kowaslki-Glikman: contribution to these Proceedings; J. Kowaslki-Glikman and L. Smolin: Phys. Rev. D70, 065020 (2004); J. Kowaslki-Glikman: Introduction to Doubly Special Relativity, hep-th/ 0405273; J. Kowaslki-Glikman and S. Nowak: Int. J. Mod. Phys. D12, 299 (2003).

25. J. F. Donoghue: Phys. Rev. Lett. 72, 2996 (1994); J. F. Donoghue: Phys. Rev. D50, 3874 (1994).

26. D.A.R. Dalvit, F.D. Mazzitelli and C. Molina-Paris: Phys. Rev. D63, 084023 (2001).

27. G. Amelino-Camelia, J.R. Ellis, N.E. Mavromatos and D.V. Nanopoulos: Int. J. Mod. Phys. A12, 607 (1997); J.R. Ellis, N.E. Mavromatos and D.V. Nanopoulos: Gen. Rel. Grav. 31, 1257 (1999); J.R. Ellis, N.E. Mavromatos and D.V. Nanopoulos: Phys. Rev. D62, 084019 (2000) J.R. Ellis, K. Farakos, N.E. Mavromatos, V.A. Mitsou and D.V. Nanopoulos, Astrophys. J. 535, 139 (2000); J.R. Ellis, N.E. Mavromatos, D.V. Nanopoulos and G. Volkov: Gen. Rel. Grav. 32, 1777 (2000); E. Gravanis and N.E. Mavromatos: JHEP 0206, 019 (2002).

28. J.R. Ellis, N.E. Mavromatos and D.V. Nanopoulos: Gen. Rel. Grav. 32, 127 (2000); J. Ellis, N. E. Mavromatos and D. V. Nanopoulos: Phys. Rev. D61, $027503(2000)$

29. J.R. Ellis, N.E. Mavromatos, D.V. Nanopoulos and A.S. Sakharov: Nature (London) 428, 386 (2004).

30. R. Gambini and J. Pullin: Phys. Rev. D59, 124021 (1999).

31. J. Alfaro, H.A. Morales-Técotl and L.F. Urrutia: Phys. Rev. Lett. 84, 2318 (2000)

32. J. Alfaro, H.A. Morales-Técotl and L.F. Urrutia: Phys. Rev. D65, 103509 (2002).

33. J. Alfaro, H.A. Morales-Técotl and L.F. Urrutia: Phys. Rev. D66, 124006 (2002).

34. L.F. Urrutia: Mod. Phys. Lett. A17, 943 (2002); L.F. Urrutia: Loop Quantum Gravity Induced Modifications to Particle Dynamics. In: AIP Conference Proceedings 670A, ed by U. Cotti, M. Mondragón and G. Tavares-Velasco (American Institute of Physics, New York 2003) pp 289-297.

35. H. Sahlmann, T. Thiemann and O. Winkler: Nucl. Phys. B606, 401 (2001); H. Sahlmann and T. Thiemann: Towards the QFT on curved spacetime limit of QRG. I: A general scheme, gr-qc/0207030 H. Sahlmann and T. Thiemann: Towards the QFT on curved spacetime limit of QRG.II: A concrete implementation, gr-qc/0207031

36. T. Padmanabhan: Phys. Rev. D57, 6206 (1998); K. Srinivasan, L. Sriramkumar and T. Padmanabhan: Phys. Rev. D58, 044009 (1998); S. Shankaranarayanan and T. Padmanabhan: Int. J. Mod. Phys. D10, 351 (2001).

37. C. Chryssomalakos and E. Okon: Int. J. Mod. Phys D13, 2003 (2004); A. Corichi and D. Sudarsky: New quantum gravity phenomenology, gr-qc/0503078

38. P. Huet and M. Peskin: Nucl Phys. B434, 3 (1995); J.R. Ellis, J. López, N.E. Mavromatos and D.V. Nanopoulos: Phys. Rev. D53, 3846 (1996).

39. S. Coleman and S.L. Glashow, Phys. Rev. D59, 116008 (1999).

40. G. Amelino-Camelia, J.R. Ellis, N.E. Mavromatos, D.V. Nanopoulos and S. Sarkar: Nature (London) 393, 763 (1998). 
41. J.R. Ellis, N.E. Mavromatos, D.V. Nanopoulos and A.S. Sakharov: Astron. Astrophys. 402, 409 (2002); J.R. Ellis, N.E. Mavromatos and A.S. Sakarov: Astropart. Phys. 20, 669 (2004).

42. S.E. Boggs, C.B. Wunderer, K. Hurley and W. Coburn: Astrophys. J. 611, L77 (2004)

43. E. Waxman and J. Bahcall: Phys. Rev. Letts. 78, 2292 (1997); E. Waxman: Nucl. Phys. (Proc. Supl) 91, 494 (2000); E. Waxman: Nucl. Phys. (Proc. Supl) 87, 345 (2000).

44. M. Vietri: Phys. Rev. Letts. 80, 3690 (1998).

45. R. Bluhm: contribution to these Proceedings; R. Bluhm: QED Test of Lorentz Symmetry, hep-ph/041149; R. Bluhm, V.A. Kostelecký, C.D. Lane and N. Russell, Phys. Rev. Lett. 88, 090801 (2002); R. Bluhm and V.A. Kostelecký, Phys. Rev. Lett. 84, 1381 (2000).

46. R.J. Gleiser and C. N. Kozameh: Phys. Rev. D64, 083007 (2001).

47. T. Kifune: Astrophys. J. Lett. 518, L21 (1999).

48. G. Amelino-Camelia and T. Piran: Phys. Rev. D64, 036005 (2001).

49. G. Amelino-Camelia: Phys.Lett. B 528, 181 (2002).

50. J. Alfaro and G. Palma: Phys. Rev. D65, 103516 (2002); J. Alfaro and G. Palma: Phys. Rev. D67, 083003 (2003).

51. T.J. Konopka and S.A. Major: New J. Phys. 4, 57 (2002).

52. R. Montemayor and L.F. Urrutia: Phys. Lett. B. 606, 86 (2005); R. Montemayor and L.F. Urrutia: Radiation in Lorentz Violating Electrodynamics. In: Gravitation and Cosmology, AIP Conference Proceedings 758, ed by A. Macías, C. Lämmerzhal and D. Nuñez (American Institute of Physics, New York, 2005) pp 81-89; R. Montemayor and L.F. Urrutia: Synchrotron radiation in Lorentz-violating Electrodynamics: the Myers-Pospelov model, hep-ph/0505135

53. B. González, S. A. Martínez, R. Montemayor and L.F. Urrutia: Lorentz Violating Electrodynamics, hep-ph/0505145

54. H. Vucetich: Testing Lorentz invariance violation in quantum gravity theories, gr-qc/0502093 D. Mattingly: Modern test of Lorentz invariance, gr-qc/0502097. T. Jacobson, S. Liberati and D. Mattingly: Lorentz violation at high energy: concepts, phenomena and astrophysical constraints, astro-ph/0505277

55. T. Thiemann: Introduction to Modern Canonical Quantum General Relativity, gr-qc/0110034 A. Pérez: Introduction to Loop Quantm Gravity and Spin Foams, grqc/0409061.

56. C. Rovelli: Helv. Phys. Acta 69, 582 (1996); C. Rovelli: Phys. Rev. Lett.77, 3288 (1996); A. Ashtekar, J. Baez, A. Corichi and K. Krasnov: Phys. Rev. Lett. 80, 904 (1998)

57. M. Bojowald: Phys. Rev. Lett. 86, 5227 (2001); For a recent review see for example M. Bojowald and H.A. Morales-Técotl: Cosmological applications of loop quantum gravity. In: Proceedings of 5th Mexican School on Gravitation and Mathematical Physics: The Early Universe and Observational Cosmology (DGFM 2002), Lect. Notes Phys. 646, ed by N. Bretón, J.L. Cervantes-Cota and M. Salgado (Springer, Berlin 2004) pp 421-462.

58. C. Rovelli and L. Smolin: Nucl. Phys. B442, 593 (1995) ; Erratum-ibid B456, 753 (1995); A. Ashtekar and J. Lewandowsky: Class. Quant. Grav. 14, A55 (1997); A. Ashtekar and J. Lewandowsky: Adv. Theor. Math. Phys. 1, 388 
(1998); A. Ashtekar, A. Corichi and J. Zapata: Class. quant. Grav. 15, 2955 (1998).

59. A. Ashtekar: Phys. Rev. Letts. 57, 2244 (1987); F. Barbero: Phys. Rev. D51, 5507 (1995); F. Barbero: Phys. Rev. D51, 5498 (1995).

60. G. Immirzi: Class. Quant. Grav. 14, L177 (1997).

61. T. Thiemann: Phys. Letts. B380, 257 (1996).

62. T. Thiemann: Class. Quan. Grav. 15, 1281 (1998); T. Thiemann: Class. Quant. Grav. 15, 839 (1998).

63. M. Varadarajan and J.A. Zapata: Class. Quant. Grav. 17, 4085 (2000); M. Varadarajan: Phys. Rev. D61, 104001 (2000); T. Thiemann: Class. Quant. Grav. 18, 2025 (2001); T. Thiemann and O. Winkler: Class. Quant. Grav. 18, 2561 (2001); T. Thiemann and O. Winkler: Class. Quant. Grav. 18, 4629 (2001); T. Thiemann and O. Winkler: Class. Quant. Grav. 18, 4997 (2001); H. Sahlmann, T. Thiemann, O. Winkler: JHEP 0105, 021 (2001); A. Ashtekar and J. Lewandowski: Class. Quant. Grav. 18, L117 (2001); A. Ashtekar, S. Fairhurst and J.L. Willis: Class. Quant. Grav. 20, 1031 (2003); A. Ashtekar, L. Bombelli and A. Corichi: Semiclassical states for constrained systems, gr-qc/0504052

64. J. Alfaro, H.A. Morales-Técotl, M. Reyes and L.F. Urrutia: J. Phys. A: Math. Gen. 36, 12097 (2003).

65. G. Lambiase: Mod. Phys. Lett. A18, 23 (2003).

66. G.L. Fogli, E. Lisi, A. Marrone and G. Scioscia: Phys. Rev. D60, 053006 (1999); R. Brustein, D. Eichler and S. Foffa: Phys. Rev. D65, 105006 (2002).

67. C. H. Lineweaver et al.: Astrophys J. 470, 38 (1996).

68. D. Sudarsky, L. Urrutia and H. Vucetich: Phys. Rev. Lett. 89, 231301 (2002).

69. J. D. Prestage et al.: Phys. Rev. Letts. 54, 2387 (1985); S. K. Lamoreaux et al.: Phys. Rev. Letts. 57, 3125 (1986); S. K. Lamoreaux et al.: Phys. Rev. A 39, 1082 (1989); C. J. Berglund et al.: Phys. Rev. Lett. 751879 (1995); D. F. Phillips et al.: Phys. Rev D 63111101 (2001); R. Walsworth: contribution to these Proceedings.

70. T.E. Chupp et al.: Phys. Rev. Letts. 63, 1541 (1989).

71. D. Bear et al.: Phys. Rev. Letts. 855038 (2000).

72. V.A. Kostelecký and C.D. Lane: J. Math. Phys. 40, 6245 (1999); V.A. Kostelecký and R. Lehnert: Phys. Rev. D63, 065008 (2001); D. Colladay and P. McDonald: J. Math. Phys. 43, 3554 (2002); For a review see for example V.A. Kostelecký: Topics in Lorentz and CPT violations. In: The Role of Neutrinos, Strings, Gravity and Variable Cosmological Constant in Elementary Particle Physics, ed by B.N. Kursunoglu, S.L. Mintz and A. Perlmutter (Kluwer, New York 2001) pp 57-68.

73. D. Sudarsky, L. Urrutia and H. Vucetich: Phys. Rev. D68, 024010 (2003).

74. J. Collins, A. Perez, D. Sudarsky, L. Urrutia and H. Vucetich: Phys. Rev. Lett. 93, 191301 (2004).

75. J. Collins, A. Perez, D. Sudarsky, L. Urrutia and H. Vucetich: unpublished.

76. S.G. Nibbelink and M. Pospelov: Phys. Rev. Letts. 94, 081601 (2005) ; P. Jain and J.P. Ralston: Supersymmetry and the Lorentz fine tuning problem, hep-th/0502106 P.A. Bolokov, S.G. Nibbelink and M. Pospelov: Lorentz violating supersymmetric quantum electrodynamics, hep-ph/0505029

77. J. Alfaro: Phys. Rev. Lett 94, 221302 (2005); J. Alfaro: LIV dimensional regularization and quantum gravity effects in the standard model, hep-th/0501129. 
J. Alfaro: Quantum gravity induced Lorentz invariance violation in the Standard Model: hadrons, hep-th/0505228

78. G. Amelino-Camelia: New J. Phys. 6, 188 (2004); T. Jacobson, S. Liberati and D. Mattingly: Comments on "Improved limit on quantum-spacetime modifications of Lorentz symmetry from observations on gamma-ray blazers", gr-qc/0212002 v2.

79. W. Coburn and S. E. Boggs: Nature (London) 423, 415 (2003); W. Coburn and S. E. Boggs: Statistical uncertainty in the re-analysis of polarization in GRB021206, astro-ph/0310515

80. R. E. Rutledge and D. B. Fox: Mon. Not. Roy. Astron. Soc. 350, 1272 (2004); C. Wigger et al.: Astrophys. J. 613, 1088 (2004).

81. J. Schwinger: Phys. Rev. 75, 1912 (1949); J. Schwinger: On the radiation by electrons in a betatron, transcribed by M. A. Furman. In: A Quantum Legacy, ed by K. Milton, (World Scientific, Singapore, 2000).

82. J. Schwinger, L.L. DeRaad, Jr., K.A. Milton and Wu-yang Tsai: Classical Electrodynamics, (Westview Press, Boulder, 1998).

83. J. Schwinger, W-Y. Tsai and T. Erber: Ann . Phys. (NY) 96, 303 (1976).

84. See for example T. Erber: Rev. Mod. Phys. 38, 626 (1966).

85. T. Erber, D. White and H.G. Latal: Acta Phys. Austr. 45, 29 (1976).

86. S.M. Carroll, G.B. Field and R. Jackiw: Phys. Rev. D41, 1231 (1990); V.A. Kostelecký and M. Mewes, Phys. Rev. Lett. 87, 251304 (2001); R. Lehnert and R. Potting: Phys. Rev. Lett. 93, 110402 (2004); Q.G. Bailey, and V.A. Kostelecky: Phys. Rev. D70, 076006 (2004).

87. A.M. Atoyan and F.A. Aharonian: Mon. Not. R. Astron. Soc. 278, 525 (1996); P.L. Marsden, F.C. Gillett, F.E. Jennings, J.P. Emerson, T. de Jong and F.N. Olnon: Astrophys. J. 278, L29 (1984).

88. F.A. Aharonian: New Astron. 5, 377 (2000).

89. A. Konopelko, A. Mastichiadis, J. Kirk, O.C. de Jager and F.W. Stecker: Astrophys. J. 597, 851 (2003).

90. E. Nakar, T. Piran and E. Waxman: JCAP 0310, 005 (2003).

91. E. Waxman: Nature (London) 423, 388 (2003).

92. W. Hajdas et al.: Time resolved annalysis of the Gamma Ray Bursts with the RHESSI satellite. In: Particle acceleration in astrophysical objects, (http://www.oa.uj.edu.pl/konferencje/proc0.html).

93. P. Castorina, A. Iorio and D. Zappala: Phys. Rev. D69, 065008 (2004).

94. G. B. Rybicki and A.P. Lightman: Radiative Processes in Astrophysics, (John Wiley and Sons, New York, 1979); A. L. Peratt: Physics of the Plasma Universe, (Springer Verlag, New York 1991)

95. S. A. Martínez, R. Montemayor and L. F. Urrutia: Reality and causality in LIV radiation, in preparation. 